\title{
Characteristic classes of foliations via SAYD-twisted cocycles
}

\author{
Bahram Rangipour and Serkan Sütlü*
}

\begin{abstract}
We find the first non trivial "SAYD-twisted" cyclic cocycle over the groupoid action algebra under the symmetry of the affine linear transformations of the Euclidian space. We apply the cocycle to construct a characteristic map by which we transfer the characteristic classes of transversely orientable foliations into the cyclic cohomology of the groupoid action algebra. In codimension 1, our result matches with the (only explicit) computation done by Connes-Moscovici. We carry out the explicit computation in codimension 2 to present the transverse fundamental class, the Godbillon-Vey class, and the other four residual classes as cyclic cocycles on the groupoid action algebra.
\end{abstract}

Mathematics Subject Classification (2010). 16T05, 16E40, 19D55, 16W30.

Keywords. Connes-Moscovici Hopf algebras, Hopf cyclic cohomology, cyclic cohomology, Weil algebra, characteristic classes of foliations.

\section{Contents}

1 Introduction . . . . . . . . . . . . . . . . 966

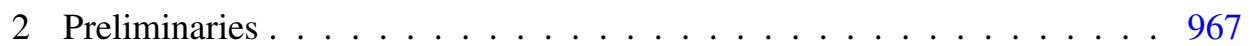

2.1 Hopf-cyclic cohomology with coefficients . . . . . . . . . . . 967

2.2 Lie algebra $($ co)homology . . . . . . . . . . . . . . . 970

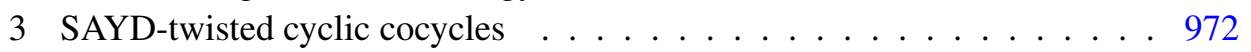

3.1 Equivariant Hopf-cyclic cohomology . . . . . . . . . . . . . 972

3.2 Equivariant characteristic map . . . . . . . . . . . . . 975

3.3 Equivariant characteristic map for $\mathcal{H}_{n} \ldots \ldots$. . . . . . . . . . 977

3.4 A SAYD-twisted cyclic cocycle in codimension 1 . . . . . . . . . 980

3.5 A SAYD-twisted cyclic cocycle in codimension 2 . . . . . . . . . . 981

4 The characteristic map with coefficients . . . . . . . . . . . . . . 987

4.1 The characteristic map in codimension 1 . . . . . . . . . . . . 988

4.2 The characteristic map in codimension 2 . . . . . . . . . . . . 989

References . . . . . . . . . . . . . . . . . . 997

\footnotetext{
${ }^{*}$ The project is partially supported by NCN-grant 2011/01/B/ST1/06474.
} 


\section{Introduction}

Following Connes-Moscovici [2], let $\mathcal{A}:=C_{c}^{\infty}\left(F^{+}\right) \rtimes \Gamma$. Here $F^{+}$is the oriented frame bundle over $\mathbb{R}^{n}$, and $\Gamma$ is a discrete subgroup of $\operatorname{Diff}^{+}\left(\mathbb{R}^{n}\right)$, the group of orientation preserving diffeomorphisms of $\mathbb{R}^{n}$.

For an arbitrary $\Gamma$, the cyclic cohomology of $\mathcal{A}$ is not known [1, Sect. III.2]. However, there is a map, even in the level of complexes,

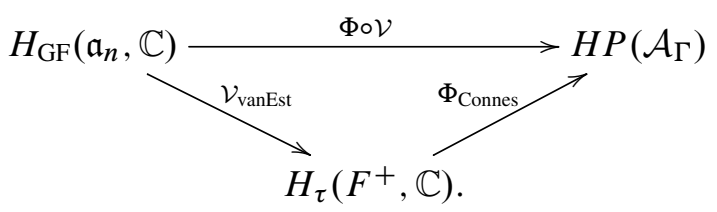

from the Gelfand-Fuks cohomology of $\mathfrak{a}_{n}$, the Lie algebra of formal vector fields on $\mathbb{R}^{n}$, that factors through the twisted cohomology computed by the Bott bicomplex [1, Prop. III.2.11]. One notes that a basis for the Gelfand-Fuks classes are known, [4], but it is difficult to transfer them to the cyclic cohomology of $\mathcal{A}$. The reader is referred to [3] for a complete account of this computation in codimension 1.

The Hopf-cyclic cohomology, invented by Connes-Moscovici [2], made it possible to have a very explicit characteristic map

$$
\chi_{\tau}: H P\left(\mathcal{H}, \mathbb{C}_{\delta}\right) \rightarrow H P(\mathcal{A}),
$$

where $\mathcal{H}:=\mathcal{H}_{n}$ is the Connes-Moscovici Hopf algebra of codimension $n, \mathbb{C}_{\delta}$ is the canonical one dimensional SAYD module over $\mathcal{H}$, and $\tau$ is the canonical trace on $\mathcal{A}$.

Although (1.2) has a simple presentation on the level of complexes, and $H P\left(\mathcal{H}, \mathbb{C}_{\delta}\right)$ is canonically isomorphic to $H_{\mathrm{GF}}\left(\mathfrak{a}_{n}, \mathbb{C}\right)$, the isomorphism is not easy to present $[2,11]$. Therefore, from the point of view of (1.2), the obstacle to transfer the characteristic classes of transversely orientable foliations to the cyclic cohomology of $\mathcal{A}$ is to find a basis of the representatives of the Hopf-cyclic classes of $\mathcal{H}$. There is an intensive ongoing study $[9,10,11]$ on the Hopf-cyclic cohomology of the geometric bicrossed product Hopf algebras such as $\mathcal{H}$.

In the present paper we develop a new characteristic map, whose source is the Hopf-cyclic cohomology of $\mathcal{K}:=U\left(g \ell_{n}\right)$, the enveloping algebra of the general linear Lie algebra $g \ell_{n}$. Since the Hopf algebra $\mathcal{K}$ is not as sophisticated as $\mathcal{H}$, one expects, by the conservation of work, a more sophisticated characteristic map and SAYD module than $\chi_{\tau}$ and $\mathbb{C}_{\delta}$ respectively.

In fact, the first step of our mission was taken in [13], where the authors showed that the truncated Weil algebra is a Hopf-cyclic complex. As a result, the characteristic classes of transversely orientable foliations can be calculated from $H C(\mathcal{K}, V)$. Here $V:=S\left(g \ell_{n}^{*}\right)_{[2 n]}$, the algebra of $n$-truncated polynomials on $g \ell_{n}$, is a canonical and nontrivial SAYD module over $\mathcal{K}$. 
The backbone of this new characteristic map is a SAYD twisted cyclic $n$-cocycle $\varphi \in C_{\mathcal{K}}^{n}(\mathcal{A}, V)$ by which we apply the cup product introduced in [8] by Khalkhali and the first named author. We use the explicit formula derived in [12] to compute the characteristic classes of foliations as cyclic cocycles in $H C(\mathcal{A})$ via

$$
\chi_{\varphi}: H C^{\bullet}(\mathcal{K}, V) \rightarrow H C^{\bullet+n}(\mathcal{A}), \quad \chi_{\varphi}(x)=x \cup \varphi .
$$

In order to test our method we first carry out the computation for codimension 1 and observe that our result matches with the classes obtained by Connes-Moscovici in [3]. The result of [11] shows that the amount of work in codimension 2 is not comparable with that of codimension 1 . However, we completely determine the representatives of all classes in $H C(\mathcal{K}, V)$, in addition to an explicit formula for $\varphi \in H C_{\mathcal{K}}^{2}(\mathcal{A}, V)$. Then (1.3) yields our desired cyclic cocycles in $H C(\mathcal{A})$.

Throughout the paper, all vector spaces and their tensor products are over $\mathbb{C}$ unless otherwise is specified. We use the Sweedler's notation for comultiplication and coaction. We denote the comultiplication of a coalgebra $C$ by $\Delta: C \rightarrow C \otimes C$ and its action on $c \in C$ by $\Delta(c)=c_{(1)} \otimes c_{(2)}$. The image of $v \in V$ under a left coaction $\nabla: V \rightarrow C \otimes V$ is denoted by $\nabla(v)=v_{<-1>} \otimes v_{<0>}$, summation suppressed. By the coassociativity, we simply write $\Delta\left(c_{(1)}\right) \otimes c_{(2)}=c_{(1)} \otimes \Delta\left(c_{(2)}\right)=$ $c_{(1)} \otimes c_{(2)} \otimes c_{(3)}$. Unless stated otherwise, a Lie algebra $\mathfrak{g}$ is finite dimensional with a basis $\left\{X_{i} \mid 1 \leq i \leq n\right\}$ and a dual basis $\left\{\theta^{i} \mid 1 \leq i \leq n\right\}$. In particular, for $\mathfrak{g}=g \ell_{n}$ we use $\left\{Y_{i}^{j} \mid 1 \leq i, j \leq n\right\}$ for a basis and $\left\{\theta_{j}^{i} \mid 1 \leq i, j \leq n\right\}$ for a dual basis. We denote the Weil algebra of $\mathfrak{g}$ by $W(\mathfrak{g})$, and $W(\mathfrak{g})_{[2 n]}$ stands for the $n$-truncated Weil algebra of $\mathfrak{g}$. We denote the Kronecker symbol by $\delta_{j}^{i}$. We also adopt the Einstein summation convention on the repeating indices unless otherwise is stated. Finally, for the sake of simplicity we use

$$
B_{\sigma(1)} \otimes \cdots \otimes B_{\sigma(q)}:=\sum_{\sigma \in S_{q}} \operatorname{sgn}(\sigma) B_{\sigma(1)} \otimes \cdots \otimes B_{\sigma(q)}
$$

for any set of objects $\left\{B_{1}, \ldots, B_{q}\right\}$. Here $S_{q}$ is the group of all permutations on $q$ objects and $\operatorname{sgn}(\sigma)$ stands for the signature of $\sigma$.

\section{Preliminaries}

In this section we recall the definition of Hopf-cyclic cohomology, the ConnesMoscovici characteristic map, and basics of the cyclic cohomology of Lie algebras.

2.1. Hopf-cyclic cohomology with coefficients. Let $H$ be a Hopf algebra, $\delta$ : $H \rightarrow \mathbb{C}$ be a character, and $\sigma$ be a group-like element $\sigma \in H$. The pair $(\delta, \sigma)$ is called a modular pair in involution (MPI for short) if

$$
\delta(\sigma)=1 \text { and } S_{\delta}^{2}=\operatorname{Ad}_{\sigma},
$$


where $\operatorname{Ad}_{\sigma}(h)=\sigma h \sigma^{-1}$, for any $h \in H$, and $S_{\delta}$ is defined by

$$
S_{\delta}(h)=\delta\left(h_{(1)}\right) S\left(h_{(2)}\right), \quad h \in H .
$$

A vector space $V$ is called a right-left stable-anti-Yetter-Drinfeld module (SAYD for short) over $H$ if it is a right $H$-module, a left $H$-comodule, and for any $v \in V$ and $h \in H$

$$
\nabla(v \cdot h)=S\left(h_{(3)}\right) v_{<-1>} h_{(1)} \otimes v_{<0>} \cdot h_{(2)}, \quad v_{<0>} \cdot v_{<-1>}=v .
$$

Using $\delta$ and $\sigma$ one endows ${ }^{\sigma} \mathbb{C}_{\delta}:=\mathbb{C}$ with a right module and left comodule structures over $H$.

Let $V$ be a right-left SAYD module over $H$ and $C$ an $H$-module coalgebra, i.e. $\Delta(h(c))=h_{(1)}\left(c_{(1)}\right) \otimes h_{(2)}\left(c_{(2)}\right)$ and $\varepsilon(h(c))=\varepsilon(h) \varepsilon(c)$, for $h \in H$ and $c \in C$.

We have the cocyclic module

$$
\begin{gathered}
C_{H}(C, V):=\bigoplus_{q \geq 0} C_{H}^{q}(C, V), \quad C_{H}^{q}(C, V):=V \otimes_{H} C^{\otimes q+1} \\
\mathfrak{d}_{i}: C_{H}^{q}(C, V) \rightarrow C_{H}^{q+1}(C, V), \quad 0 \leq i \leq q+1 \\
\mathfrak{d}_{i}\left(v \otimes_{H} c^{0} \otimes \cdots \otimes c^{q}\right)=v \otimes_{H} c^{0} \otimes \cdots \otimes \Delta\left(c^{i}\right) \otimes \cdots \otimes c^{q}, \\
\mathfrak{d}_{q+1}\left(v \otimes_{H} c^{0} \otimes \cdots \otimes c^{q}\right)= \\
v_{<0>} \otimes_{H} c^{0}(2) \otimes c^{1} \otimes \cdots \otimes c^{q} \otimes v_{<-1>}\left(c^{0}(1)\right), \\
\mathfrak{s}_{j}: C_{H}^{q}(C, V) \rightarrow C_{H}^{q-1}(C, V), \quad 0 \leq j \leq q-1 \\
\mathfrak{s}_{j}\left(v \otimes_{H} c^{0} \otimes \cdots \otimes h^{q}\right)=v \otimes_{H} c^{0} \otimes \cdots \otimes \varepsilon\left(c^{j+1}\right) \otimes \cdots \otimes c^{q}, \\
\mathfrak{t}_{q}: C_{H}^{q}(C, V) \rightarrow C_{H}^{q}(C, V), \\
\mathfrak{t}_{q}\left(v \otimes_{H} c^{0} \otimes \cdots \otimes c^{q}\right)=v_{<0>} \otimes_{H} c^{1} \otimes \cdots \otimes c^{q} \otimes v_{<-1>}\left(c^{0}\right) .
\end{gathered}
$$

Using the above operators one defines the Hochschild coboundary $b$ and the Connes boundary operator $B$,

$$
\begin{aligned}
& b: C_{H}^{q}(C, V) \rightarrow C_{H}^{q+1}(C, V), \quad b:=\sum_{i=0}^{q+1}(-1)^{i} \mathfrak{d}_{i}, \\
& B: C_{H}^{q}(C, V) \rightarrow C_{H}^{q-1}(C, V), \quad B:=\left(\sum_{i=0}^{q}(-1)^{q i} \mathfrak{t}^{i}\right) \mathfrak{s}_{q-1} \mathfrak{t} .
\end{aligned}
$$

We denote the cyclic cohomology of $C_{H}(C, V)$ by $H C_{H}(C, V)$.

For $C=H$, with its multiplication as the action, the map

$$
\begin{aligned}
\mathcal{J}: V \otimes_{H} H^{\otimes(n+1)} \rightarrow V \otimes H^{\otimes n}, \\
\quad \mathcal{J}\left(v \otimes_{H} h^{0} \otimes \cdots \otimes h^{n}\right)=v h_{(1)}^{0} \otimes S\left(h_{(2)}\right) \cdot\left(h^{1} \otimes \cdots \otimes h^{n}\right) .
\end{aligned}
$$


identifies the standard Hopf-cyclic complex (2.4) with

$$
C(H, V):=\bigoplus_{q \geq 0} C^{q}(H, V), \quad C^{q}(H, V):=V \otimes H^{\otimes q} .
$$

$$
\begin{aligned}
\mathfrak{d}_{0}\left(v \otimes h^{1} \otimes \cdots \otimes h^{q}\right) & =v \otimes 1 \otimes h^{1} \otimes \cdots \otimes h^{q}, \\
\mathfrak{d}_{i}\left(v \otimes h^{1} \otimes \cdots \otimes h^{q}\right) & =v \otimes h^{1} \otimes \cdots \otimes h^{i}{ }_{(1)} \otimes h^{i}(2) \otimes \cdots \otimes h^{q}, \\
\mathfrak{d}_{q+1}\left(v \otimes h^{1} \otimes \cdots \otimes h^{q}\right) & =v_{<0>} \otimes h^{1} \otimes \cdots \otimes h^{q} \otimes v_{<-1>}, \\
\mathfrak{s}_{j}\left(v \otimes h^{1} \otimes \cdots \otimes h^{q}\right) & =v \otimes h^{1} \otimes \cdots \otimes \varepsilon\left(h^{j+1}\right) \otimes \cdots \otimes h^{q}, \\
\mathfrak{t}\left(v \otimes h^{1} \otimes \cdots \otimes h^{q}\right) & =v_{<0>} h^{1}{ }_{(1)} \otimes S\left(h_{(2)}^{1}\right) \cdot\left(h^{2} \otimes \cdots \otimes h^{q} \otimes v_{<-1>}\right) .
\end{aligned}
$$

Let $A$ be a $H$-module algebra, that is, a (left) $H$-module and

$$
h(a b)=h_{(1)}(a) h_{(2)}(b), \quad h\left(1_{A}\right)=\varepsilon(h) 1_{A}, \quad \forall h \in H, a \in A .
$$

Then one endows $V \otimes A^{\otimes n+1}$ with the action of $H$ as

$$
\left(v \otimes a^{0} \otimes \cdots \otimes a^{q}\right) \cdot h=v h_{(1)} \otimes S\left(h_{(q+2)}\right) a^{0} \otimes \cdots \otimes S\left(h_{(2)}\right) a^{q} .
$$

We set

$$
C_{H}^{n}(A, V)=\operatorname{Hom}_{H}\left(V \otimes A^{\otimes n+1}, \mathbb{C}\right)
$$

as the space of $H$-linear maps. It is checked in [5] that for any $v \otimes \tilde{a}:=v \otimes a^{0} \otimes$ $\cdots \otimes a^{n+1} \in V \otimes A^{\otimes n+2}$ the morphisms

$$
\begin{aligned}
\left(\partial_{i} \varphi\right)(v \otimes \tilde{a}) & =\varphi\left(v \otimes a^{0} \otimes \ldots \otimes a^{i} a^{i+1} \otimes \ldots \otimes a^{n+1}\right), \quad 0 \leq i \leq n, \\
\left(\partial_{n+1} \varphi\right)(v \otimes \tilde{a}) & =\varphi\left(v_{<0>} \otimes\left(S^{-1}\left(v_{<-1>}\right) a^{n+1}\right) a^{0} \otimes a^{1} \otimes \ldots \otimes a^{n}\right), \\
\left(\sigma_{i} \varphi\right)(v \otimes \tilde{a}) & =\varphi\left(v \otimes a^{0} \otimes \ldots \otimes a^{i} \otimes 1 \otimes \ldots \otimes a^{n-1}\right), \quad 0 \leq i \leq n-1, \\
(\tau \varphi)(v \otimes \tilde{a}) & =\varphi\left(v_{<0>} \otimes\left(S^{-1}\left(v_{<-1>}\right) a^{n}\right) \otimes a^{0} \otimes \ldots \otimes a^{n-1}\right)
\end{aligned}
$$

define a cocyclic module structure on $C_{H}(A, V)$, whose cyclic cohomology is denoted by $H C_{H}(A, V)$.

One uses $H C_{H}(H, V)$ and $H C_{H}(A, V)$ to define a cup product

$$
H C_{H}^{p}(A, V) \otimes H C_{H}^{q}(H, V) \rightarrow H C^{p+q}(A),
$$

whose definition can be found in $[12,8]$.

As the simplest example, one notes that the cup product with the 0 -cocycle $\tau \in$ $C_{H}^{0}\left(A,{ }^{\sigma} \mathbb{C}_{\delta}\right)$ defines the Connes-Moscovici characteristic map [2, 3],

$$
\begin{aligned}
& \chi_{\tau}: H C^{n}\left(H,{ }^{\sigma} \mathbb{C}_{\delta}\right) \rightarrow H C^{n}(A) \\
& \quad \chi_{\tau}\left(h^{1} \otimes \cdots \otimes h^{n}\right)\left(a^{0} \otimes \cdots \otimes a^{n}\right)=\tau\left(a^{0} h^{1}\left(a^{1}\right) \ldots h^{n}\left(a^{n}\right)\right) .
\end{aligned}
$$


2.2. Lie algebra (co)homology. In this subsection we summarize our work in [13] on the cyclic cohomology of Lie algebras with coefficients in SAYD modules.

Let $\mathfrak{g}$ be a Lie algebra and $V$ be a right $\mathfrak{g}$-module. Lets recall the Lie algebra homology complex

$$
C(\mathfrak{g}, V)=\bigoplus_{q \geq 0} C_{q}(\mathfrak{g}, V), \quad C_{q}(\mathfrak{g}, V):=\wedge^{q} \mathfrak{g} \otimes V,
$$

with the Chevalley-Eilenberg boundary map

$$
\begin{aligned}
& \cdots \stackrel{\partial_{\mathrm{CE}}}{\longrightarrow} C_{2}(\mathfrak{g}, V) \stackrel{\partial_{\mathrm{CE}}}{\longrightarrow} C_{1}(\mathfrak{g}, V) \stackrel{\partial_{\mathrm{CE}}}{\longrightarrow} V, \\
& \partial_{\mathrm{CE}}\left(X_{0} \wedge \cdots \wedge X_{q-1} \otimes v\right)=\sum_{i=0}^{q-1}(-1)^{i} X_{0} \wedge \cdots \wedge \widehat{X}_{i} \wedge \cdots \wedge X_{q-1} \otimes v \cdot X_{i} \\
& \quad+\sum_{0 \leq i<j \leq q-1}(-1)^{i+j}\left[X_{i}, X_{j}\right] \wedge X_{0} \wedge \cdots \wedge \widehat{X}_{i} \wedge \cdots \wedge \widehat{X}_{j} \wedge \cdots \wedge X_{q-1} \otimes v .
\end{aligned}
$$

The homology of the complex $\left(C(\mathfrak{g}, V), \partial_{\mathrm{CE}}\right)$ is called the Lie algebra homology of $\mathfrak{g}$ with coefficients in $V$, and it is denoted by $H_{\bullet}(\mathfrak{g}, V)$. In a dual fashion, one defines the Lie algebra cohomology complex

$$
W(\mathfrak{g}, V)=\bigoplus_{q \geq 0} W^{q}(\mathfrak{g}, V), \quad W^{q}(\mathfrak{g}, V)=\operatorname{Hom}\left(\wedge^{q} \mathfrak{g}, V\right),
$$

where $\operatorname{Hom}\left(\wedge^{q} \mathfrak{g}, V\right)$ is the vector space of all alternating linear maps on $\mathfrak{g}^{\otimes q}$ with values in $V$. The Chevalley-Eilenberg coboundary

$$
V \stackrel{d_{\mathrm{CE}}}{\longrightarrow} W^{1}(\mathfrak{g}, V) \stackrel{d_{\mathrm{CE}}}{\longrightarrow} W^{2}(\mathfrak{g}, V) \stackrel{d_{\mathrm{CE}}}{\longrightarrow} \cdots,
$$

is defined by

$$
\begin{array}{r}
d_{\mathrm{CE}}(\alpha)\left(X_{0}, \ldots, X_{q}\right)=\sum_{0 \leq i<j \leq q}(-1)^{i+j} \alpha\left(\left[X_{i}, X_{j}\right], X_{0} \ldots \widehat{X}_{i}, \ldots, \widehat{X}_{j}, \ldots, X_{q}\right) \\
+\sum_{i=0}^{q}(-1)^{i+1} \alpha\left(X_{0}, \ldots, \widehat{X}_{i}, \ldots X_{q}\right) \cdot X_{i} .
\end{array}
$$

Alternatively, we may identify $W^{q}(\mathfrak{g}, V)$ with $\wedge^{q} \mathfrak{g}^{*} \otimes V$ and the coboundary $d_{\mathrm{CE}}$ with

$$
\begin{gathered}
d_{\mathrm{CE}}(v)=-\theta^{i} \otimes v \cdot X_{i}, \quad d_{\mathrm{CE}}(\beta \otimes v)=d_{\mathrm{dR}}(\beta) \otimes v-\theta^{i} \wedge \beta \otimes v \cdot X_{i}, \\
d_{\mathrm{dR}}: \wedge^{p} \mathfrak{g}^{*} \rightarrow \wedge^{p+1} \mathfrak{g}^{*}, \quad d_{\mathrm{dR}}\left(\theta^{i}\right)=-\frac{1}{2} C_{j k}^{i} \theta^{j} \wedge \theta^{k}
\end{gathered}
$$


The cohomology of the complex $\left(W(\mathfrak{g}, V), d_{\mathrm{CE}}\right)$, the Lie algebra cohomology of $\mathfrak{g}$ with coefficients in $V$, is denoted by $H^{\bullet}(\mathfrak{g}, V)$.

We are particularly interested in the SAYD modules over the universal enveloping algebra $U(\mathfrak{g})$ of a Lie algebra $\mathfrak{g}$, and by [13], such SAYD modules are obtained from the SAYD modules over the Lie algebra $\mathfrak{g}$.

Definition 2.1 ([13]). A vector space $V$ is a left comodule over the Lie algebra $\mathfrak{g}$ if there is a linear map

$$
\nabla_{\mathfrak{g}}: V \rightarrow \mathfrak{g} \otimes V, \quad \nabla_{\mathfrak{g}}(v)=v_{[-1]} \otimes v_{[0]}
$$

such that

$$
v_{[-2]} \wedge v_{[-1]} \otimes v_{[0]}=0,
$$

where

$$
v_{[-2]} \otimes v_{[-1]} \otimes v_{[0]}=v_{[-1]} \otimes\left(v_{[0]}\right)_{[-1]} \otimes\left(v_{[0]}\right)_{[0]} .
$$

It is clear that left $\mathfrak{g}$-comodules and right $S\left(\mathfrak{g}^{*}\right)$-modules are identical.

Definition 2.2 ([13]). Let $V$ be a right module and left comodule over a Lie algebra $\mathfrak{g}$. We call $V$ a right-left anti-Yetter-Drinfeld module (AYD module) over $\mathfrak{g}$ if

$$
\nabla_{\mathfrak{g}}(v \cdot X)=v_{[-1]} \otimes v_{[0]} \cdot X+\left[v_{[-1]}, X\right] \otimes v_{[0]} .
$$

Moreover, $V$ is called stable if

$$
v_{[0]} \cdot v_{[-1]}=0 .
$$

Finally, $V$ is said to be unimodular stable if $V_{-\delta}$ is stable,where $\delta:=\operatorname{Tr} \circ$ ad $: \mathfrak{g} \rightarrow$ $\mathbb{C}$, and $V_{-\delta}$ is the deformation of $V$ via

$$
v \triangleleft X:=v \cdot X-\delta(X) v .
$$

Example 2.3. The truncated polynomial algebra $V=S\left(\mathfrak{g}^{*}\right)_{2 n]}$, of a Lie algebra $\mathfrak{g}$, is a unimodular SAYD module over $\mathfrak{g}$ with the coadjoint action and the Koszul coaction defined by

$$
\nabla_{K}: V \rightarrow \mathfrak{g} \otimes V, \quad \nabla_{K}(v)=\sum_{i=1}^{n} X_{i} \otimes v \theta^{i}
$$

Next, by using SAYD modules, we generalize the Lie algebra (co)homology complexes. Let us start with the Lie algebra homology by introducing the complex

$$
C(\mathfrak{g}, V)=\bigoplus_{i \geq 0} \wedge^{i} \mathfrak{g} \otimes V, \quad \partial:=\partial_{\mathrm{CE}}+\partial_{\mathrm{K}}
$$

with the Chevalley-Eilenberg boundary and the Koszul coboundary

$$
\partial_{\mathrm{K}}: C_{n}(\mathfrak{g}, V) \rightarrow C_{n+1}(\mathfrak{g}, V), \quad \partial_{\mathrm{K}}(e \otimes v)=v_{[-1]} \wedge e \otimes v_{[0]} .
$$


Applying the Poincaré duality, see [13, Prop. 4.4], we obtain

$$
W(\mathfrak{g}, V)=\bigoplus_{i \geq 0} \wedge^{i} \mathfrak{g}^{*} \otimes V, \quad d:=d_{\mathrm{CE}}+d_{\mathrm{K}}
$$

where $d_{\mathrm{CE}}: W^{n}(\mathfrak{g}, V) \rightarrow W^{n+1}(\mathfrak{g}, V)$ is the Chevalley-Eilenberg coboundary and

$$
d_{\mathrm{K}}: W^{n}(\mathfrak{g}, V) \rightarrow W^{n-1}(\mathfrak{g}, V), \quad d_{\mathrm{K}}(\alpha \otimes v)=\iota\left(v_{[-1]}\right)(\alpha) \otimes v_{[0]}
$$

is the Koszul boundary, $\iota(X)$ being the contraction by $X$.

In particular, we recover the (truncated) Weil algebra [13]:

$$
W\left(\mathfrak{g}, S\left(\mathfrak{g}^{*}\right)\right)=W(\mathfrak{g}), \quad W\left(\mathfrak{g}, S\left(\mathfrak{g}^{*}\right)_{[2 n]}\right)=W(\mathfrak{g})_{[2 n]} .
$$

\section{SAYD-twisted cyclic cocycles}

In this section we fix $K$ to be a cocommutative Hopf subalgebra of a Hopf algebra $H$, $A$ an $H$-module algebra, and $V$ a SAYD module over $K$. We aim to develop a machinery to produce cyclic cocycles in $H C_{K}(A, V)$. In the first subsection we introduce equivariant Hopf-cyclic cohomology $H C_{K}(H, V, N)$, where $N$ is a SAYD module over $H$. In the second subsection we construct a cup product

$$
H C_{K}^{p}(H, V, N) \otimes H C_{H}^{q}(A, N) \rightarrow H C_{K}^{p+q}(A, V) .
$$

In the third and fourth subsections we apply the results of the first two subsections to produce a nontrivial SAYD-twisted cyclic cocycle over the groupoid action algebra under the symmetry of the general linear Lie algebra with coefficients in the truncated polynomials on this Lie algebra.

3.1. Equivariant Hopf-cyclic cohomology. For a SAYD module $N$ over $H$ and a module-comodule $V$ over $K$ we define the graded space

$$
\begin{aligned}
& C_{K}(H, V, N)=\bigoplus_{q \geq 0} C_{K}^{q}(H, V, N), \\
& \mathcal{C}^{q}:=C_{K}^{q}(H, V, N):=\operatorname{Hom}_{K}\left(V, N \otimes_{H} H^{\otimes q+1}\right) .
\end{aligned}
$$

More precisely, $\phi \in \mathcal{C}^{q}$ if and only if for any $u \in K$ and any $v \in V$

$$
\phi(v \cdot u)=\phi(v) \cdot u,
$$

where the right action of $K$ on $N \otimes_{H} H^{\otimes q+1}$ is the usual diagonal action, i.e.

$$
\left(n \otimes_{H} h^{0} \otimes \cdots \otimes h^{q}\right) \cdot u=n \otimes_{H} h^{0} u_{(1)} \otimes \cdots \otimes h^{q} u_{(q+1)} .
$$


For $\phi \in C_{K}^{q}(H, V, N)$ and $v \in V$, we use the notation

$$
\phi(v)=\phi(v)^{[-1]} \otimes_{H} \phi(v)^{[0]} \otimes \cdots \otimes \phi(v)^{[q]} .
$$

Let us define the morphisms $d_{i}: \mathcal{C}^{q} \rightarrow \mathcal{C}^{q+1}, s_{j}: \mathcal{C}^{q} \rightarrow \mathcal{C}^{q-1}$, and $t_{q}: \mathcal{C}^{q} \rightarrow \mathcal{C}^{q}$ as

$$
\begin{aligned}
d_{i}(\phi)(v) & =\mathfrak{d}_{i}(\phi(v)), \quad 0 \leq i \leq q \\
d_{q+1}(\phi)(v) & =\mathfrak{d}_{q+1}\left(\phi\left(v_{<0>}\right)\right) \triangleleft S\left(v_{<-1>}\right), \\
s_{j}(\phi)(v) & =\mathfrak{s}_{j}(\phi(v)), \quad 0 \leq j \leq q-1, \\
t_{q}(\phi)(v) & =\mathfrak{t}_{q}\left(\phi\left(v_{<0>}\right)\right) \triangleleft S\left(v_{<-1>}\right),
\end{aligned}
$$

where the right action $\triangleleft$ of $K$ on $N \otimes_{H} H^{\otimes q+1}$ is defined by

$$
\left(n \otimes_{H} h^{0} \otimes \cdots \otimes h^{q}\right) \triangleleft u=n \otimes_{H} h^{0} \otimes \cdots \otimes h^{q-1} \otimes h^{q} u,
$$

and the morphisms $\mathfrak{d}_{i}, \mathfrak{s}_{j}$ and $\mathfrak{t}$ are the usual morphisms of the cocyclic module $C_{H}(H, N)$ defined in (2.5), (2.6) and (2.7).

Theorem 3.1. If $V$ and $N$ are $S A Y D$ modules over $K$ and $H$ respectively, then the morphisms $d_{i}, s_{j}$ and $t$ define a cocyclic module structure on $C_{K}(H, V, N)$.

Proof. Let us prove that the morphisms $d_{i}, s_{j}$, and $t$ are well-defined. To this end, it suffices to check that $t, d_{0}$, and $s_{n}$ are well-defined as the other morphisms are made of these three. For $d_{0}$ and $s_{n}$ the task is obvious as $\Delta: H \rightarrow H \otimes H$ and $\varepsilon: H \rightarrow \mathbb{C}$ are multiplicative respectively. As for $t$, we have

$$
\begin{aligned}
& t(\phi)(v \cdot y)=\tau\left(\phi\left((v \cdot y)_{<0>}\right)\right) \triangleleft S\left((v \cdot y)_{<-1>}\right) \\
& =\tau\left(\phi\left(v_{<0>}\right) \cdot y_{(2)}\right) \triangleleft S\left(y_{(3)}\right) S\left(v_{<-1>}\right) y_{(1)} \\
& =\tau\left(\phi(v)^{[-1]} \otimes_{H} \phi(v)^{[0]} y_{(2)} \otimes \ldots\right. \\
& \left.\ldots \otimes \phi(v)^{[q]} y_{(q+2)}\right) \triangleleft S\left(y_{(q+3)}\right) S\left(v_{<-1>}\right) y_{(1)} \\
& =\phi(v)^{[-1]}<0>\otimes_{H} \phi(v)^{[1]} y_{(3)} \otimes \ldots
\end{aligned}
$$

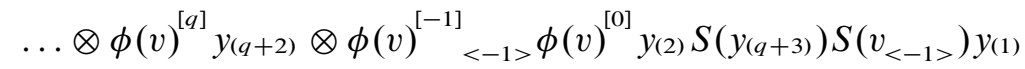

$$
\begin{aligned}
& =\phi(v)^{[-1]}<0>\otimes_{H} \phi(v)^{[1]} y_{(1)} \otimes \ldots \\
& \ldots \otimes \phi(v)^{[q]} y_{(q)} \otimes \phi(v)_{<-1>}^{[-1]} \phi(v)^{[0]} S\left(v_{<-1>}\right) y_{(q+1)} \\
& =t(\phi(v)) \cdot y \text {. }
\end{aligned}
$$

In the second and the sixth equalities we use the fact that $K$ is cocommutative. 
Let us next show that $C_{K}(H, V, N)$ is a cocyclic module, that is,

$$
\begin{gathered}
d_{j} d_{i}=d_{i} d_{j-1}, i<j, \quad s_{j} s_{i}=s_{i} s_{j+1}, i \leq j \\
s_{j} d_{i}= \begin{cases}d_{i} s_{j-1} & \text { if } i<j \\
\operatorname{Id}_{q} & \text { if } i=j \text { or } i=j+1 ; \\
d_{i-1} s_{j} & \text { if } i>j+1\end{cases} \\
t_{q+1} d_{i}=d_{i-1} t_{q}, \quad 1 \leq i \leq q+1, \quad t_{q+1} d_{0}=d_{q+1} \\
t_{q-1} s_{i}=s_{i-1} t_{q}, \quad 1 \leq i \leq q-1, \quad t_{q} s_{0}=s_{q-1} t_{q}^{2} \\
t_{q}^{q+1}=\operatorname{Id}_{q} .
\end{gathered}
$$

The equalities (3.8), (3.9) and (3.11) follow directly from their counterparts for the operators $\mathfrak{d}_{i}, \mathfrak{s}_{j}$ and $\mathfrak{t}$.

We check (3.10) for $i=q+1$. Indeed,

$$
\begin{aligned}
& t_{q+1}\left(d_{q+1}(\phi)\right)(v)=\mathfrak{t}_{q+1}\left(d_{q+1}(\phi)\left(v_{<0>}\right)\right) \triangleleft S\left(\left(v_{<-1>}\right)\right) \\
& =\mathfrak{t}_{q+1}\left(\mathfrak{d}_{q+1}\left(\phi\left(v_{<0><0>}\right)\right) \triangleleft S\left(v_{<0><-1>}\right)\right) \triangleleft S\left(v_{<-1>}\right) \\
& =\mathfrak{t}_{q+1}\left(\mathfrak{d}_{q+1}\left(\phi\left(v_{<0>}\right)\right) \triangleleft S\left(v_{<-1>}\right)\right) \triangleleft S\left(v_{<-2>}\right) \\
& =\mathfrak{t}_{q+1}\left(\phi\left(v_{<0>}\right)^{[-1]}<0>\otimes_{H} \phi\left(v_{<0>}\right)^{[0]}{ }_{(2)} \otimes \phi\left(v_{<0>}\right)^{[1]} \otimes \ldots\right.
\end{aligned}
$$

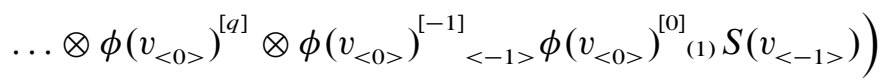

$$
\begin{aligned}
& \triangleleft S\left(v_{<-2>}\right) \\
& =\phi\left(v_{<0>}\right)^{[-1]}<0>\otimes_{H} \phi\left(v_{<0>}\right)^{[1]} \otimes \cdots \otimes \phi\left(v_{<0>}\right)^{[q]}
\end{aligned}
$$

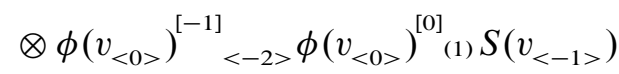

$$
\begin{aligned}
& \otimes \phi\left(v_{<0>}\right)^{[-1]}<-1>\phi\left(v_{<0>}\right)^{[0]}(2) S\left(v_{<-2>}\right) \\
& =\phi\left(v_{<0>}\right)^{[-1]}<0>\otimes_{H} \phi\left(v_{<0>}\right)^{[1]} \otimes \cdots \otimes \phi\left(v_{<0>}\right)^{[q]} \\
& \otimes \Delta\left(\phi\left(v_{<0>}\right)_{<-1>}^{[-1]} \phi\left(v_{<0>}\right)^{[0]} S\left(v_{<-1>}\right)\right) \\
& =\mathfrak{d}_{q}\left(\left[\phi\left(v_{<0>}\right)^{[-1]}<0>\otimes_{H} \phi\left(v_{<0>}\right)^{[1]} \otimes \cdots \otimes \phi\left(v_{<0>}\right)^{[q]}\right.\right. \\
& \left.\left.\otimes \phi\left(v_{<0>}\right)^{[-1]}<-1>\phi\left(v_{<0>}\right)^{[0]}\right] \triangleleft S\left(v_{<-1>}\right)\right) \\
& =\mathfrak{d}_{q}\left(\mathfrak{t}_{q}\left(\phi\left(v_{<0>}\right)\right) \triangleleft S\left(v_{<-1>}\right)\right)=d_{q}\left(t_{q}(\phi)\right)(v) .
\end{aligned}
$$

As for (3.12), a simple calculation yields

$$
\begin{aligned}
& t_{q}^{q+1}(\phi)(v)=\phi\left(v_{<0>}\right)^{[-1]}<0>\otimes_{H} \phi\left(v_{<0>}\right)_{<-q-1>}^{[-1]} \phi\left(v_{<0>}\right)^{[0]} S\left(v_{<-1>}\right) \otimes \ldots \\
& \ldots \otimes \phi\left(v_{<0>}\right)_{<-1>}^{[-1]} \phi\left(v_{<0>}\right)^{[q]} S\left(v_{<-q-1>}\right) \otimes \ldots
\end{aligned}
$$




$$
\begin{aligned}
& =\phi\left(v_{<0>}\right)^{[-1]}<0>\phi\left(v_{<0>}\right)^{[-1]}<-1>\otimes_{H} \phi\left(v_{<0>}\right)^{[0]} S\left(v_{<-1>}\right) \ldots \\
& \ldots \otimes \phi\left(v_{<0>}\right)^{[q]} S\left(v_{<-q-1>}\right) \\
& =\phi\left(v_{<0>}\right)^{[-1]} \otimes_{H} \phi\left(v_{<0>}\right)^{[0]} S\left(v_{<-1>}\right) \otimes \cdots \otimes \phi\left(v_{<0>}\right)^{[q]} S\left(v_{<-q-1>}\right) \\
& =\phi\left(v_{<0>}\right) \cdot S\left(v_{<-1>}\right)=\phi\left(v_{<0>} \cdot S\left(v_{<-1>}\right)\right)=\phi(v),
\end{aligned}
$$

where the last equality follows from [6, Lemma 4.9].

We denote the cyclic cohomology of $C_{K}(H, V, N)$ by $H C_{K}(H, V, N)$. One notes that by taking $K=\mathbb{C}$ and $V=\mathbb{C}$ the usual Hopf-cyclic cohomology $H C(H, N)$ is recovered.

3.2. Equivariant characteristic map. Let $V$ and $N$ be SAYD modules over $K$ and $H$ respectively. We define

$$
\begin{aligned}
& \Psi: C_{K}^{q}(H, V, N) \otimes C_{H}^{q}(A, N) \longrightarrow C_{K}^{q}(A, V) \\
& \begin{array}{l}
\Psi(\phi \otimes \psi)\left(v \otimes a_{0} \otimes \cdots \otimes a_{q}\right) \\
\quad=\psi\left(\phi(v)^{[-1]} \otimes \phi(v)^{[0]}\left(a_{0}\right) \otimes \phi(v)^{[1]}\left(a_{1}\right) \otimes \cdots \otimes \phi(v)^{[q]}\left(a_{q}\right)\right) .
\end{array}
\end{aligned}
$$

One may check that $\Psi$ is a map of cocyclic modules, where on the left hand side we consider the product of two cocyclic modules. This is enough to produce a generalization of the cup product in Hopf-cyclic cohomology [8, 12].

We define a bicocyclic module by tensoring (2.13) and (3.1). The bigraded module in the bidegree $(p, q)$ is then defined by

$$
\mathcal{C}^{p, q}:=\operatorname{Hom}_{K}\left(V, N \otimes H H^{\otimes p+1}\right) \otimes \operatorname{Hom}_{H}\left(A^{\otimes q+1}, N\right)
$$

with the horizontal structure $\vec{\partial}_{i}=\mathfrak{d}_{i} \otimes \mathrm{Id}, \vec{\sigma}_{j}=\mathfrak{s} \otimes$ Id and $\vec{\tau}=\mathfrak{t} \otimes \mathrm{Id}$, and the vertical structure $\uparrow \partial_{i}=\operatorname{Id} \otimes \partial_{i}, \uparrow \sigma_{j}=\operatorname{Id} \otimes \sigma_{j}$ and $\uparrow \tau=\operatorname{Id} \otimes \tau$. Obviously $\left(\mathcal{C}^{\bullet}, \bullet, \vec{\partial}, \vec{\sigma}, \vec{\tau}, \uparrow \partial, \uparrow \sigma, \uparrow \tau\right)$ defines a bicocyclic module.

Now let us define the map

$$
\begin{aligned}
& \Psi: \mathcal{D}^{q} \rightarrow \operatorname{Hom}_{K}\left(V \otimes A^{\otimes q+1}, \mathbb{C}\right) \\
& \Psi(\phi \otimes \psi)\left(v \otimes a_{0} \otimes \cdots \otimes a_{q}\right) \\
& \quad=\psi\left(\phi(v)^{[-1]} \otimes \phi(v)^{[0]}\left(a_{0}\right) \otimes \phi(v)^{[1]}\left(a_{1}\right) \otimes \cdots \otimes \phi(v)^{[q]}\left(a_{q}\right)\right),
\end{aligned}
$$

where $\mathcal{D}^{\bullet}$ denotes the diagonal of the bicocyclic module $\mathcal{C}^{\bullet \bullet} \bullet$. It is a cocyclic module whose $q$ th component is $\mathcal{C}^{q, q}$ and its cocyclic structure maps are $\partial_{i}:=\vec{\partial}_{i} \circ \uparrow \partial_{i}$, $\sigma_{j}:=\vec{\sigma}_{j} \circ \uparrow \sigma_{j}$, and $\tau:=\vec{\tau} \circ \uparrow \tau$.

Proposition 3.2. The map $\Psi$, defined in (3.17), is a map of cocyclic modules. 
Proof. Let us first show that $\Psi$ is well-defined. Indeed, using the fact that $\phi$ is $K$ linear, we see that

$$
\begin{aligned}
& \Psi(\phi \otimes \psi)\left(v k_{(1)} \otimes S\left(k_{(q+2)}\right)\left(a^{0}\right) \otimes \cdots \otimes S\left(k_{(2)}\right)\left(a^{q}\right)\right) \\
& \quad=\psi\left(\phi\left(v k_{(1)}\right)^{[-1]} \otimes \phi\left(v k_{(1)}\right)^{[0]} S\left(k_{(q+2)}\right)\left(a^{0}\right) \otimes \cdots \otimes \phi\left(v k_{(1)}\right)^{[q]} S\left(k_{(2)}\right)\left(a^{q}\right)\right) \\
& \quad=\psi\left(\phi(v)^{[-1]} \otimes \phi(v)^{[0]} k_{(1)} S\left(k_{(2 q+2)}\right)\left(a^{0}\right) \otimes \cdots \otimes \phi(v)^{[q]} k_{(q+1)} S\left(k_{(q+2)}\right)\left(a^{q}\right)\right) \\
& \quad=\varepsilon(k) \Psi(\phi \otimes \psi)\left(v \otimes a^{0} \otimes \cdots \otimes a^{q}\right) .
\end{aligned}
$$

Next, we show that $\Psi$ commutes with the cocyclic structures. To this end, it suffices to show the commutativity of $\Psi$ with zeroth coface, the last codegeneracy and the cyclic operator. We check it only for the cyclic operators and leave the rest to the reader.

$$
\begin{aligned}
& \tau(\Psi(\phi \otimes \psi))\left(v \otimes a^{0} \otimes \cdots \otimes a^{q}\right) \\
&=\Psi(\phi \otimes \psi)\left(v_{<0>} \otimes S^{-1}\left(v_{<-1>}\right)\left(a^{q}\right) \otimes a^{0} \otimes \cdots \otimes a^{q-1}\right) \\
&=\psi\left(\phi\left(v_{<0>}\right)^{[-1]} \otimes \phi\left(v_{<0>}\right)^{[0]} S^{-1}\left(v_{<-1>}\right)\left(a^{q}\right)\right. \\
& \otimes \phi\left(v_{<0>}\right)^{[1]}\left(a^{1}\right) \otimes \cdots \otimes \phi\left(v_{<0>}\right)^{[q]}\left(a^{q}\right) .
\end{aligned}
$$

On the other hand we have

$$
\begin{aligned}
& \Psi(\mathfrak{t} \phi \otimes \tau \psi)\left(v \otimes a^{0} \otimes \cdots \otimes a^{q}\right) \\
& =\tau \psi\left(\mathfrak{t} \phi(v)^{[-1]} \otimes \mathfrak{t} \phi(v)^{[0]}\left(a^{0}\right) \otimes \cdots \otimes \mathfrak{t} \phi(v)^{[q]}\left(a^{q}\right)\right)
\end{aligned}
$$

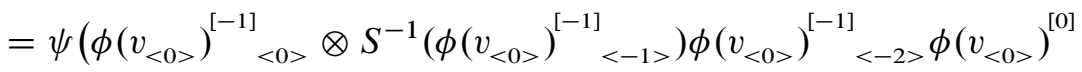

$$
\begin{aligned}
& \left.S\left(v_{<-1>}\right)\left(a^{q}\right) \otimes \phi(v)^{[1]}\left(a^{0}\right) \otimes \cdots \otimes \phi(v)^{[q]}\left(a^{q-1}\right)\right) \\
& =\psi\left(\phi\left(v_{<0>}\right)^{[-1]} \otimes \phi\left(v_{<0>}\right)^{[0]} S\left(v_{<-1>}\right)\left(a^{q}\right)\right. \\
& \otimes \phi\left(v_{<0>}\right)^{[1]}\left(a^{1}\right) \otimes \cdots \otimes \phi\left(v_{<0>}\right)^{[q]}\left(a^{q}\right) .
\end{aligned}
$$

The proof is complete in view of $S=S^{-1}$.

Theorem 3.3. Assume that $K$ is a cocommutative Hopf subalgebra of a Hopf algebra $H, A$ is a $H$-module algebra, and $V$ and $N$ are SAYD modules over $K$ and $H$ respectively. Then the map $\Psi$ defines a cup product

$$
H C_{K}^{p}(H, V, N) \otimes H C_{H}^{q}(A, N) \rightarrow H C_{K}^{p+q}(A, V) .
$$

Proof. Let $[\phi] \in H C_{K}^{p}(H, V, N)$ and $[\psi] \in H C_{H}^{q}(A, N)$. Without loss of generality we assume that $\phi$ and $\psi$ are, respectively, cyclic cocycles horizontally and vertically. This implies that $\phi \otimes \psi$ is a $(b, B)$ cocycle of degree $p+q$ in total complex of $\mathcal{C}^{\bullet, \bullet}$. On the other hand, by the cyclic Eilenberg-Zilber theorem [7], 
the total complex of $\mathcal{C}^{\bullet}, \bullet$ is quasi-isomorphic with $\mathcal{D}^{\bullet}$ via the Alexander-Whitney map $A W$. So, $A W(\phi \otimes \psi)$ is a $(b, B)$ cocycle in $\mathcal{D}^{\bullet}$. Since $\Psi$ is cyclic, we conclude that $\Psi(A W(\phi \otimes \psi))$ defines a class in $H C_{K}^{p+q}(A, V)$.

One notes that for $K=\mathbb{C}$ and $V=\mathbb{C}$, the trivial SAYD module over $K$, the above cup product becomes the one defined in $[8,12]$.

3.3. Equivariant characteristic map for $\mathcal{H}_{n}$. In this subsection we apply the equivariant characteristic map of Subsection 3.2 to produce the desired cyclic cocycle on the groupoid action algebra $\mathcal{A}:=C_{c}^{\infty}\left(F^{+}\right) \rtimes \Gamma$.

Let us first recall the Connes-Moscovici Hopf algebra $\mathcal{H}:=\mathcal{H}_{n}$ from [2, 3]. To this end, let $\mathfrak{h}_{n}$ be the Lie algebra generated by

$$
\left\{X_{k}, Y_{i}^{j}, \delta_{j k \ell_{1} \ldots \ell_{r}}^{i} \mid i, j, k, \ell_{1} \ldots \ell_{r}=1, \ldots, n, r \in \mathbb{N}\right\}
$$

with relations

$$
\begin{gathered}
{\left[Y_{i}^{j}, Y_{k}^{\ell}\right]=\delta_{k}^{j} Y_{i}^{\ell}-\delta_{i}^{\ell} Y_{k}^{j}, \quad\left[Y_{i}^{j}, X_{k}\right]=\delta_{k}^{j} X_{i}, \quad\left[X_{k}, X_{\ell}\right]=0,} \\
\delta_{j k \ell_{1} \ldots \ell_{r}}^{i}=\left[X_{\ell_{r}}, \ldots\left[X_{\ell_{1}}, \delta_{j k}^{i}\right] \ldots\right], \quad\left[\delta_{j k \ell_{1} \ldots \ell_{r}}^{i}, \delta_{j^{\prime} k^{\prime} \ell_{1}^{\prime} \ldots \ell_{r}^{\prime}}^{i^{\prime}}\right]=0, \\
{\left[Y_{p}^{q}, \delta_{j_{1} j_{2} j_{3} \ldots j_{r}}^{i}\right]=\sum_{s=1}^{r} \delta_{j_{s}}^{q} \delta_{j_{1} j_{2} j_{3} \ldots j_{s-1} p j_{s+1} \ldots j_{r}}^{i}-\delta_{p}^{i} \delta_{j_{1} j_{2} j_{3} \ldots j_{r}}^{q},} \\
\delta_{j k \ell_{1} \ldots \ell_{r}}^{i}=\delta_{j k \ell_{\pi(1)} \ldots \ell_{\pi(r)}, \quad \forall \pi \in S_{r} .}
\end{gathered}
$$

As an algebra, $\mathcal{H}$ is $U\left(\mathfrak{h}_{n}\right)$ modulo the (Bianchi-type) identities

$$
\delta_{j \ell k}^{i}-\delta_{j k \ell}^{i}=\delta_{j k}^{s} \delta_{s \ell}^{i}-\delta_{j \ell}^{s} \delta_{s k}^{i} .
$$

The coalgebra structure of $\mathcal{H}$ is defined by a Leibniz rule that makes $\mathcal{A}$ an $\mathcal{H}$-module algebra.

In order to describe the action of $\mathcal{H}$ explicitly, let us first identify $F^{+}$with $\mathbb{R}^{n} \rtimes \mathrm{GL}^{+}(n, \mathbb{R})$ and use the local coordinates $(x, y) \in F^{+}$. A typical element of the algebra $\mathcal{A}$ is a finite sum $\sum_{i} f_{i} U_{\phi_{i}}^{*}$, where $U_{\phi_{i}}^{*}$ stands for $\phi_{i}^{-1} \in \Gamma$ and $f_{i} \in C_{c}^{\infty}\left(F^{+}\right)$. The elements of $\mathcal{H}$ then act as

$$
\begin{gathered}
X_{k}=y_{k}^{\mu} \frac{\partial}{\partial x^{\mu}}, \quad X_{k}\left(f U_{\phi}^{*}\right):=X_{k}(f) U_{\phi}^{*}, \\
Y_{i}^{j}=y_{i}^{\mu} \frac{\partial}{\partial y_{\mu}^{j}}, \quad Y_{i}^{j}\left(f U_{\phi}^{*}\right):=Y_{i}^{j}(f) U_{\phi}^{*}, \\
\delta_{j k \ell_{1} \ldots \ell_{r}}^{i}\left(f U_{\phi}^{*}\right)=\gamma_{j k \ell_{1} \ldots \ell_{r}}^{i}(\phi) f U_{\phi}^{*},
\end{gathered}
$$

where

$$
\begin{aligned}
\gamma_{j k \ell_{1} \ldots \ell_{r}}^{i}(\phi) & =X_{\ell_{r}} \cdots X_{\ell_{1}}\left(\gamma_{j k}^{i}(\phi)\right), \\
\gamma_{j k}^{i}(\phi)(x, y) & =\left(y^{-1} \cdot \phi^{\prime}(x)^{-1} \cdot \partial_{\mu} \phi^{\prime}(x) \cdot y\right)_{j}^{i} y_{k}^{\mu} .
\end{aligned}
$$


Therefore, for any $a, b \in \mathcal{A}$ we have the Leibniz rule

$$
\begin{aligned}
& Y_{i}^{j}(a b)=Y_{i}^{j}(a) b+a Y_{i}^{j}(b), \\
& X_{k}(a b)=X_{k}(a) b+a X_{k}(b)+\delta_{j k}^{i}(a) Y_{i}^{j}(b), \\
& \delta_{j k}^{i}(a b)=\delta_{j k}^{i}(a) b+a \delta_{j k}^{i}(b) .
\end{aligned}
$$

Accordingly,

$$
\begin{aligned}
& \Delta\left(Y_{i}^{j}\right)=Y_{i}^{j} \otimes 1+1 \otimes Y_{i}^{j}, \\
& \Delta\left(\delta_{j k}^{i}\right)=\delta_{j k}^{i} \otimes 1+1 \otimes \delta_{j k}^{i}, \\
& \Delta\left(X_{k}\right)=X_{k} \otimes 1+1 \otimes X_{k}+\delta_{j k}^{i} \otimes Y_{i}^{j} .
\end{aligned}
$$

For simplicity, we will also employ the notation

$$
\delta^{a}{ }_{k \ell_{1} \ldots \ell_{r}}:=\delta_{j k \ell_{1} \ldots \ell_{r}}^{i}, \quad Y_{a}:=Y_{i}^{j} \quad a=\left(\begin{array}{l}
i \\
j
\end{array}\right) .
$$

Let us also set $\mathfrak{g}_{0}:=g \ell_{n}, V=S\left(\mathfrak{g}_{0}^{*}\right)_{[2 n]}$ with the canonical SAYD module structure over $\mathcal{K}:=U\left(\mathfrak{g}_{0}\right)$ as recalled in (2.3), and $N=\mathbb{C}_{\delta}$ the SAYD module over $\mathcal{H}$ where $\delta: \mathcal{H} \rightarrow \mathbb{C}$ is the character defined on the generators by

$$
\delta\left(Y_{i}^{j}\right)=\delta_{i}^{j}, \quad \delta\left(X_{k}\right)=\delta\left(\delta_{j k \ell_{1} \ldots \ell_{r}}^{i}\right)=0, \quad 1 \leq i, j, k, l_{t} \leq n .
$$

Applying the cup product (3.18) with the canonical trace $\tau \in C_{\mathcal{H}}^{0}(\mathcal{A}, N)$, [2], we get the characteristic map

$$
\begin{aligned}
& \chi_{\tau}^{\mathrm{eq}}: H C_{\mathcal{K}}^{q}(\mathcal{H}, V, N) \longrightarrow H C_{\mathcal{K}}^{q}(\mathcal{A}, V) \\
& \chi_{\tau}^{\mathrm{eq}}(\phi)\left(v \otimes a_{0} \otimes \cdots \otimes a_{q}\right)=\tau\left(\phi(v)^{[0]}\left(a_{0}\right) \cdots \phi(v)^{[q]}\left(a_{q}\right)\right),
\end{aligned}
$$

as a mechanism to obtain "SAYD-twisted" cyclic cocycles. We conclude this section by the identification of $C_{\mathcal{K}}^{q}(\mathcal{K}, V, N)$ with $\left(V^{*} \otimes N \otimes \mathcal{H}^{\otimes q}\right)^{\mathfrak{g}_{0}}$, where $V^{*}=\operatorname{Hom}_{\mathbb{C}}(V, \mathbb{C})$ and $\mathfrak{g}_{0}$ acts on $V^{*} \otimes N \otimes \mathcal{H}^{\otimes q}$ via

$$
\begin{gathered}
\left(\phi \otimes \mathbf{1} \otimes h^{1} \otimes \cdots \otimes h^{q}\right) Z=-\sum_{i=1}^{q} \phi \otimes \mathbf{1} \otimes h^{1} \otimes \cdots \otimes \operatorname{ad}_{Z}\left(h^{i}\right) \otimes \cdots \otimes h^{q} \\
+\phi \otimes \delta(Z) \otimes h^{1} \otimes \cdots \otimes h^{q}+\phi \cdot Z \otimes \mathbf{1} \otimes h^{1} \otimes \cdots \otimes h^{q} .
\end{gathered}
$$

Here, the action of $\mathfrak{g}_{0}$ on $V^{*}$ is defined by $(\phi \cdot Z)(v)=-\phi(v \cdot Z)$. Accordingly, the aforementioned identification is given by the map

$$
\begin{aligned}
& \mathcal{I}:\left(V^{*} \otimes N \otimes \mathcal{H}^{\otimes q}\right)^{\mathfrak{g}_{0}} \rightarrow C_{\mathcal{K}}^{q}(\mathcal{H}, V, N) \\
& \mathcal{I}\left(\phi \otimes \mathbf{1} \otimes h^{1} \otimes \cdots \otimes h^{q}\right)(v)=\phi(v) \otimes_{\mathcal{H}} 1_{\mathcal{H}} \otimes h^{1} \otimes \cdots \otimes h^{q} .
\end{aligned}
$$


Proposition 3.4. The map $\mathcal{I}$, defined in (3.32), is an isomorphism of vector spaces.

Proof. Let us first check that $\mathcal{I}$ is well-defined. Indeed,

$$
\begin{aligned}
& \mathcal{I}\left(\phi \otimes \mathbf{1} \otimes h^{1} \otimes \cdots \otimes h^{q}\right)(v \cdot Z) \\
& =\phi(v \cdot Z) \otimes_{\mathcal{H}} 1_{\mathcal{H}} \otimes h^{1} \otimes \cdots \otimes h^{q} \\
& =-(\phi \cdot Z)(v) \otimes_{\mathcal{H}} 1_{\mathcal{H}} \otimes h^{1} \otimes \cdots \otimes h^{q} \\
& =-\sum_{i=1}^{q} \phi(v) \otimes_{\mathcal{H}} 1_{\mathcal{H}} \otimes h^{1} \otimes \cdots \otimes \operatorname{ad}_{Z}\left(h^{i}\right) \otimes \cdots \otimes h^{q} \\
& +\delta(Z) \phi(v) \otimes_{\mathcal{H}} 1_{\mathcal{H}} \otimes h^{1} \otimes \cdots \otimes h^{q} \\
& =-\sum_{i=1}^{q} \phi(v) \otimes_{\mathcal{H}} 1_{\mathcal{H}} \otimes h^{1} \otimes \cdots \otimes \operatorname{ad}_{Z}\left(h^{i}\right) \otimes \cdots \otimes h^{q} \\
& +\phi(v) \otimes_{\mathcal{H}} Z \otimes h^{1} \otimes \cdots \otimes h^{q} \\
& +\sum_{i=1}^{q} \phi(v) \otimes_{\mathcal{H}} 1_{\mathcal{H}} \otimes h^{1} \otimes \cdots \otimes Z h^{i} \otimes \cdots \otimes h^{q} \\
& =\left(\phi(v) \otimes 1_{\mathcal{H}} \otimes h^{1} \otimes \cdots \otimes h^{q}\right) \cdot Z \\
& =\left(\mathcal{I}\left(\phi \otimes \mathbf{1} \otimes h^{1} \otimes \cdots \otimes h^{q}\right)(v)\right) \cdot Z .
\end{aligned}
$$

Next, we introduce an inverse map for $\mathcal{I}$. To this end we fix a basis for $V$, say $\left\{v_{1}, \ldots, v_{m}\right\}$, with a dual basis $\left\{v^{1}, \ldots, v^{m}\right\}$ for $V^{*}$. Then,

$$
\begin{aligned}
& \mathcal{I}^{-1}: C_{\mathcal{K}}^{q}(\mathcal{H}, V, N) \rightarrow\left(V^{*} \otimes N \otimes \mathcal{H}^{\otimes q}\right)^{\mathfrak{g}_{0}} \\
& \mathcal{I}^{-1}(\phi)=\sum_{i=1}^{m} v^{i} \otimes \phi\left(v_{i}\right)^{[-1]} \delta\left(\phi\left(v_{i}\right)^{[0]}{ }_{(1)}\right) \otimes S\left(\phi\left(v_{i}\right)^{[0]}{ }_{(2)}\right) \\
& \left.\cdot\left(\phi\left(v_{i}\right)^{[1]}\right) \otimes \cdots \otimes \phi\left(v_{i}\right)^{[q]}\right)
\end{aligned}
$$

is inverse to $\mathcal{I}$, and is independent of the choice of bases.

As a result, we can transfer the cocyclic structure of $C_{\mathcal{K}}(\mathcal{H}, V, N)$ to

$$
\mathcal{E}\left(V^{*}, N, \mathcal{H}\right)^{\mathrm{inv}}:=\bigoplus_{q \geq 0} \mathcal{E}^{q}, \quad \mathcal{E}^{q}:=\left(V^{*} \otimes N \otimes \mathcal{H}^{\otimes q}\right)^{\mathfrak{g}_{0}} .
$$

This way we obtain the cocyclic structure with the cofaces

$$
\begin{aligned}
& \widetilde{\mathfrak{d}}_{i}: \mathcal{E}^{q} \longrightarrow \mathcal{E}^{q+1}, \quad 0 \leq i \leq q+1, \\
& \widetilde{\mathfrak{d}}_{0}\left(\phi \otimes \mathbf{1} \otimes h^{1} \otimes \cdots \otimes h^{q}\right)=\phi \otimes \mathbf{1} \otimes 1 \otimes h^{1} \otimes \cdots \otimes h^{q}, \\
& \widetilde{\mathfrak{d}}_{i}\left(\phi \otimes \mathbf{1} \otimes h^{1} \otimes \cdots \otimes h^{q}\right)=\phi \otimes \mathbf{1} \otimes h^{1} \otimes \cdots \otimes \Delta\left(h^{i}\right) \otimes \cdots \otimes h^{q}, \\
& \widetilde{\mathfrak{d}}_{q+1}\left(\phi \otimes \mathbf{1} \otimes h^{1} \otimes \cdots \otimes h^{q}\right)=v^{i} \phi\left(v_{i_{<0>}}\right) \otimes \mathbf{1} \otimes h^{1} \otimes \cdots \otimes h^{q} \otimes S\left(v_{i_{<-1>}}\right),
\end{aligned}
$$


the codegeneracies

$$
\begin{aligned}
& \widetilde{\mathfrak{s}}_{j}: \mathcal{E}^{q} \longrightarrow \mathcal{E}^{q-1}, \quad 0 \leq j \leq q-1 \\
& \widetilde{\mathfrak{s}}_{j}\left(\phi \otimes \mathbf{1} \otimes h^{1} \otimes \cdots \otimes h^{q}\right)=\phi \otimes \mathbf{1} \otimes h^{1} \otimes \cdots \otimes \varepsilon\left(h^{j+1}\right) \otimes \cdots \otimes h^{q},
\end{aligned}
$$

and the cyclic operator

$$
\begin{aligned}
& \widetilde{\mathfrak{t}}_{q}: \mathcal{E}^{q} \longrightarrow \mathcal{E}^{q}, \\
& \widetilde{\mathfrak{t}}_{q}\left(\phi \otimes \mathbf{1} \otimes h^{1} \otimes \cdots \otimes h^{q}\right)=v^{i} \phi\left(v_{i_{<0>}>}\right) \otimes \mathbf{1} \otimes \widetilde{S}\left(h^{1}\right) \\
& \cdot\left(h^{2} \otimes \cdots \otimes h^{q} \otimes S\left(v_{i_{<-1>}}\right)\right) .
\end{aligned}
$$

3.4. A SAYD-twisted cyclic cocycle in codimension 1. In this subsection we keep the setting of Subsection 3.3 for $n=1$. We construct an explicit equivariant cyclic 1 -cocycle $\varphi \in C_{\mathcal{K}}^{1}(\mathcal{A}, V)$.

Let $\{R\}$ be the basis for $\mathfrak{g}_{0}^{*}$ as the dual basis of $\left\{Y:=Y_{1}^{1}\right\}$ for $\mathfrak{g}_{0}$. Let also $\{1, R\}$ be the basis of $V$ and $\left\{1^{*}, S\right\}$ as the dual basis for $V^{*}$.

For $1^{*} \otimes \mathbf{1} \otimes X, S \otimes \mathbf{1} \otimes \delta_{1} \in \mathcal{E}^{1}$, we define

$$
\begin{aligned}
& \varphi_{0}, \varphi_{1}: V \otimes \mathcal{A}^{\otimes 2} \rightarrow \mathbb{C}, \\
& \varphi_{0}=\chi_{\tau}^{\mathrm{eq}}\left(1^{*} \otimes \mathbf{1} \otimes X\right), \quad \varphi_{1}=\chi_{\tau}^{\mathrm{eq}}\left(S \otimes \mathbf{1} \otimes \delta_{1}\right) .
\end{aligned}
$$

Lemma 3.5. The 1-cochain $\varphi_{0}-\varphi_{1}$ is a Hochschild 1-cocycle.

Proof. Using (3.26) and (3.27) we have

$$
\begin{array}{r}
b\left(\varphi_{0}-\varphi_{1}\right)\left((\alpha 1+\beta \theta) \otimes a_{0} \otimes a_{1} \otimes a_{2}\right) \\
=\left(\varphi_{0}-\varphi_{1}\right)\left((\alpha 1+\beta \theta) \otimes a_{0} a_{1} \otimes a_{2}\right)-\left(\varphi_{0}-\varphi_{1}\right)\left((\alpha 1+\beta \theta) \otimes a_{0} \otimes a_{1} a_{2}\right) \\
+\left(\varphi_{0}-\varphi_{1}\right)\left((\alpha 1+\beta \theta) \otimes a_{2} a_{0} \otimes a_{1}\right)-\left(\varphi_{0}-\varphi_{1}\right)\left(\alpha \theta \otimes Y\left(a_{2}\right) a_{0} \otimes a_{1}\right) \\
=\alpha \tau\left(a_{0} a_{1} X\left(a_{2}\right)\right)-\alpha \tau\left(a_{0} X\left(a_{1} a_{2}\right)\right)+\alpha \tau\left(a_{2} a_{0} X\left(a_{1}\right)\right)+\alpha \tau\left(Y\left(a_{2}\right) a_{0} \delta_{1}\left(a_{1}\right)\right) \\
-\beta \tau\left(a_{0} a_{1} \delta_{1}\left(a_{2}\right)\right)+\beta \tau\left(a_{0} \delta_{1}\left(a_{1} a_{2}\right)\right)-\beta \tau\left(a_{2} a_{0} \delta_{1}\left(a_{1}\right)\right)=0 .
\end{array}
$$

Proposition 3.6. The 1-cocycle $\varphi_{0}-\varphi_{1}$ is cyclic.

Proof. By using the $\delta$ invariancy of $\tau,(3.26)$ and (3.27) we have

$$
\begin{aligned}
& t\left(\varphi_{0}-\varphi_{1}\right)\left((\alpha 1+\beta \theta) \otimes a_{0} \otimes a_{1}\right) \\
& \quad=\left(\varphi_{0}-\varphi_{1}\right)\left((\alpha 1+\beta \theta) \otimes a_{1} \otimes a_{0}\right)-\left(\varphi_{0}-\varphi_{1}\right)\left(\alpha \theta \otimes Y\left(a_{1}\right) \otimes a_{0}\right) \\
& \quad=\alpha \tau\left(a_{1} X\left(a_{0}\right)\right)+\alpha \tau\left(Y\left(a_{1}\right) \delta_{1}\left(a_{0}\right)\right)-\beta \tau\left(a_{1} \delta_{1}\left(a_{0}\right)\right) \\
& \quad=-\alpha \tau\left(a_{0} X\left(a_{1}\right)\right)+\beta \tau\left(a_{0} \delta_{1}\left(a_{1}\right)\right) \\
& \quad=-\left(\varphi_{0}-\varphi_{1}\right)\left((\alpha 1+\beta \theta) \otimes a_{0} \otimes a_{1}\right) .
\end{aligned}
$$


3.5. A SAYD-twisted cyclic cocycle in codimension 2. As in the previous subsection, we keep the setting of Subsection 3.3 for $n=2$. We introduce an explicit cyclic 2-cocycle $\phi \in C_{\mathcal{K}}^{2}(\mathcal{A}, V)$.

Let $\left\{R_{j}^{i} \mid 1 \leq i, j \leq 2\right\}$ be the dual basis of $\mathfrak{g}_{0}$ with the pairing $\left\langle Y_{i}^{j}, R_{l}^{k}\right\rangle=$ $\delta_{k}^{j} \delta_{l}^{i}$. We take

$$
\left\{1, R_{j}^{i}, R_{l}^{k} R_{q}^{p} \mid\left(\begin{array}{c}
k \\
l
\end{array}\right) \leq\left(\begin{array}{c}
p \\
q
\end{array}\right)\right\},
$$

as a basis for $V$ which is simplified by $\left\{1, R^{a}, R^{a b} \mid a \leq b\right\}$. The dual basis for $V^{*}$ is expressed by $\left\{1^{*}, S_{a}, S_{a b} \mid a \leq b\right\}$.

We recall from [13] that the Koszul coaction (2.24) gives rise to a $\mathcal{K}$-coaction by the formula

$$
\begin{aligned}
\nabla_{K} & : V \rightarrow \mathcal{K} \otimes V, \\
\nabla_{K}(1) & =1 \otimes 1+Y_{a} \otimes R^{a}+\frac{1}{2 !} Y_{a} Y_{b} \otimes R^{a b}, \\
\nabla_{K}\left(R^{a}\right) & =1 \otimes R^{a}+Y_{b} \otimes R^{a b}, \\
\nabla_{K}\left(R^{a b}\right) & =1 \otimes R^{a b} .
\end{aligned}
$$

We decompose $V=V_{0} \oplus V_{1} \oplus V_{2}$, where $V_{0}=\mathbb{C}\langle 1\rangle, V_{1}=\mathbb{C}\left\langle R^{a}\right\rangle$, and $V_{2}=\mathbb{C}\left\langle R^{a b}\right\rangle$. Using this decomposition, any $\psi \in \operatorname{Hom}\left(V \otimes \mathcal{A}^{q+1}, \mathbb{C}\right)$ is decomposed uniquely as $\psi=\psi_{0}+\psi_{1}+\psi_{2}$ by $\psi_{i}=\left.\psi\right|_{V_{i} \otimes \mathcal{A} \otimes q+1}$.

We now consider the linear map $\psi: V \otimes \mathcal{A}^{\otimes 3} \rightarrow \mathbb{C}$ with components

$$
\begin{aligned}
\psi_{0}:=\chi_{\tau}^{\mathrm{eq}} & \left(\gamma_{1} 1^{*} \otimes \mathbf{1} \otimes X_{\sigma(1)} \otimes X_{\sigma(2)}+\gamma_{2} 1^{*} \otimes \mathbf{1} \otimes \delta^{a}{ }_{\sigma(1)} \otimes X_{\sigma(2)} Y_{a}\right. \\
& \left.+\gamma_{3} 1^{*} \otimes \mathbf{1} \otimes \delta^{a}{ }_{\sigma(1)} \delta^{b}{ }_{\sigma(2)} Y_{b} \otimes Y_{a}+\gamma_{4} 1^{*} \otimes \mathbf{1} \otimes \delta^{a}{ }_{\sigma(1) \sigma(2)} \otimes Y_{a}\right), \\
\psi_{1}:=\chi_{\tau}^{\mathrm{eq}} & \left(\beta_{1} S_{a} \otimes \mathbf{1} \otimes \delta^{a}{ }_{\sigma(1)} \otimes X_{\sigma(2)}+\beta_{2} S_{a} \otimes \mathbf{1} \otimes X_{\sigma(1)} \otimes \delta^{a}{ }_{\sigma(2)}\right. \\
& +\beta_{3} S_{a} \otimes \mathbf{1} \otimes \delta^{a}{ }_{\sigma(1)} \delta^{b}{ }_{\sigma(2)} \otimes Y_{b}+\beta_{4} S_{a} \otimes \mathbf{1} \otimes Y_{b} \otimes \delta^{a}{ }_{\sigma(1)} \delta^{b}{ }_{\sigma(2)} \\
& +\beta_{5} S_{a} \otimes \mathbf{1} \otimes \delta^{a}{ }_{\sigma(1)} Y_{b} \otimes \delta^{b}{ }_{\sigma(2)}+\beta_{6} S_{a} \otimes \mathbf{1} \otimes \delta^{b}{ }_{\sigma(1)} Y_{b} \otimes \delta^{a}{ }_{\sigma(2)} \\
& +\beta_{7} S_{a} \otimes \mathbf{1} \otimes \delta^{a}{ }_{\sigma(1)} \otimes \delta^{b}{ }_{\sigma(2)} Y_{b}+\beta_{8} S_{a} \otimes \mathbf{1} \otimes \delta^{b}{ }_{\sigma(1)} \otimes \delta^{a}{ }_{\sigma(2)} Y_{b} \\
& \left.+\beta_{9} S_{a} \otimes \mathbf{1} \otimes \Delta\left(\delta^{a}{ }_{\sigma(1) \sigma(2)}\right)\right), \\
\psi_{2}:=\chi_{\tau}^{\mathrm{eq}} & \left(\alpha_{1} S_{a b} \otimes \mathbf{1} \otimes \delta^{a}{ }_{\sigma(1)} \otimes \delta^{b}{ }_{\sigma(2)}+\alpha_{2} S_{a b} \otimes \mathbf{1} \otimes \delta^{b}{ }_{\sigma(1)} \otimes \delta^{a}{ }_{\sigma(2)}\right),
\end{aligned}
$$

Our aim is to determine the coefficients $\alpha_{i}, \beta_{j}, \gamma_{k}$, such that $\psi$ is a cyclic 2cocycle. To do so we prove a series of technical lemmas.

Lemma 3.7. For any $\alpha_{i}, \beta_{j}, \gamma_{k},(b \psi)_{2}=0$.

Proof. The result follows directly from the application of the Hochschild coboundary map and the fact that $\delta^{a}{ }_{k}$ are derivations of $\mathcal{A}$. 
On the next move, we determine $\alpha_{i}, 1 \leq i \leq 2$, in such a way that $\psi \in C_{\mathcal{K}}^{2}(\mathcal{A}, V)$ is a cyclic cocycle on $V_{2} \otimes \mathcal{A}^{\otimes 3}$.

Lemma 3.8. For $\alpha_{1}=\alpha_{2}$, we have $(\tau \psi)_{2}=\psi_{2}$.

Proof. By definition of the cyclic operator, we have

$$
\tau \psi\left(R^{a b} \otimes a_{0} \otimes a_{1} \otimes a_{2}\right)=\psi_{2}\left(R^{a b} \otimes a_{2} \otimes a_{0} \otimes a_{1}\right) .
$$

Hence, by the integration by parts property $[3,(3.4)]$,

$$
\begin{aligned}
\tau \psi\left(R^{a b} \otimes a_{0} \otimes a_{1} \otimes a_{2}\right) \\
=\alpha_{1} \tau\left(a_{0}\left(-\delta^{a}{ }_{\sigma(1)}\right) \delta^{b}{ }_{\sigma(2)}\left(a_{1}\right) a_{2}\right)+\alpha_{1} \tau\left(a_{0} \delta^{b}{ }_{\sigma(2)}\left(a_{1}\right)\left(-\delta^{a}{ }_{\sigma(1)}\right)\left(a_{2}\right)\right) \\
\quad+\alpha_{2} \tau\left(a_{0}\left(-\delta^{b}{ }_{\sigma(1)}\right) \delta^{a}{ }_{\sigma(2)}\left(a_{1}\right) a_{2}\right)+\alpha_{2} \tau\left(a_{0} \delta^{a}{ }_{\sigma(2)}\left(a_{1}\right)\left(-\delta^{b}{ }_{\sigma(1)}\right)\left(a_{2}\right)\right) \\
=\alpha_{1} \tau\left(a_{0} \delta^{b}{ }_{\sigma(1)}\left(a_{1}\right) \delta^{a}{ }_{\sigma(2)}\left(a_{2}\right)\right)+\alpha_{2} \tau\left(a_{0} \delta^{a}{ }_{\sigma(1)}\left(a_{1}\right) \delta^{b}{ }_{\sigma(2)}\left(a_{2}\right)\right) .
\end{aligned}
$$

Therefore, $(\tau \psi)_{2}=\psi_{2}$ if and only if $\alpha_{1}=\alpha_{2}$.

As a result we set

$$
\alpha_{1}=\alpha_{2}=r .
$$

On the next step, we find a constraint on $\beta_{j}$ 's such that $\psi$ is a Hochschild cocycle over $V_{1} \otimes \mathcal{A}^{\otimes 3}$.

Lemma 3.9. We have $(b \psi)_{1}=0$ if and only if

$$
\begin{array}{r}
\beta_{1}-\beta_{3}+\beta_{7}+r=0 \\
\beta_{3}+\beta_{8}+r=0 \\
-\beta_{2}-\beta_{6}+\beta_{8}=0 \\
\beta_{4}-\beta_{5}=0 \\
-\beta_{4}-\beta_{6}=0 \\
-\beta_{5}+\beta_{7}=0 .
\end{array}
$$

Proof. Recalling the Koszul coaction (3.41) in the last coface,

$$
\begin{aligned}
& b \psi\left(R^{a} \otimes a_{0} \otimes a_{1} \otimes a_{2} \otimes a_{3}\right) \\
&= \psi_{1}\left(R^{a} \otimes a_{0} a_{1} \otimes a_{2} \otimes a_{3}\right)-\psi_{1}\left(R^{a} \otimes a_{0} \otimes a_{1} a_{2} \otimes a_{3}\right) \\
&+\psi_{1}\left(R^{a} \otimes a_{0} \otimes a_{1} \otimes a_{2} a_{3}\right)-\psi_{1}\left(R^{a} \otimes a_{3} a_{0} \otimes a_{1} \otimes a_{2}\right) \\
&+\psi_{2}\left(R^{a b} \otimes Y_{b}\left(a_{3}\right) a_{0} \otimes a_{1} \otimes a_{2}\right) .
\end{aligned}
$$


Therefore, as a result of the tracial property [3, Thm. 6] and the faithfulness $[3,(3.12)]$ of the trace, we have $(b \psi)_{1}=0$ if and only if

$$
\begin{aligned}
\left(\beta_{1}-\beta_{3}\right. & \left.+\beta_{7}+r\right) \mathbf{1} \otimes \delta^{a}{ }_{\sigma(1)} \otimes \delta^{b}{ }_{\sigma(2)} \otimes Y_{b} \\
& +\left(\beta_{3}+\beta_{8}+r\right) \mathbf{1} \otimes \delta^{b}{ }_{\sigma(1)} \otimes \delta^{a}{ }_{\sigma(2)} \otimes Y_{b} \\
& +\left(-\beta_{2}-\beta_{6}+\beta_{8}\right) \mathbf{1} \otimes \delta^{b}{ }_{\sigma(1)} \otimes Y_{b} \otimes \delta^{a}{ }_{\sigma(2)} \\
& +\left(\beta_{4}-\beta_{5}\right) \mathbf{1} \otimes Y_{b} \otimes \delta^{a}{ }_{\sigma(1)} \otimes \delta^{b}{ }_{\sigma(2)} \\
& +\left(-\beta_{4}-\beta_{6}\right) \mathbf{1} \otimes Y_{b} \otimes \delta^{b}{ }_{\sigma(1)} \otimes \delta^{a}{ }_{\sigma(2)} \\
& +\left(-\beta_{5}+\beta_{7}\right) \mathbf{1} \otimes \delta^{a}{ }_{\sigma(1)} \otimes Y_{b} \otimes \delta^{b}{ }_{\sigma(2)}=0 .
\end{aligned}
$$

Accordingly, we get the system (3.45).

On the next step we determine $\beta_{j}$ 's in such a way that $\psi \in C_{\mathcal{K}}^{2}(\mathcal{A}, V)$ is a cyclic cocycle over $\left(V_{1} \oplus V_{2}\right) \otimes \mathcal{A}^{\otimes 3}$.

Lemma 3.10. We have $(\tau \psi)_{1}=\psi_{1}$ if and only if

$$
\begin{array}{ll}
\beta_{1}=\beta_{2}=-r, & \beta_{3}=\beta_{4}=\beta_{5}=-\beta_{6}=\beta_{7}=s, \\
\beta_{8}=-r-s, & \beta_{9}=\frac{1}{2} r+s .
\end{array}
$$

Proof. By the Koszul coaction, we have

$t \psi\left(R^{a} \otimes a_{0} \otimes a_{1} \otimes a_{2}\right)=\psi_{1}\left(R^{a} \otimes a_{2} \otimes a_{0} \otimes a_{1}\right)-\psi_{2}\left(R^{a b} \otimes Y_{b}\left(a_{2}\right) \otimes a_{0} \otimes a_{1}\right)$.

Accordingly,

$$
\begin{aligned}
& t \psi\left(R^{a}\right.\left.\otimes a_{0} \otimes a_{1} \otimes a_{2}\right)= \\
& \beta_{1} \tau\left(\delta^{a}{ }_{\sigma(1)}\left(a_{0}\right) X_{\sigma(2)}\left(a_{1}\right) a_{2}\right)+\beta_{2} \tau\left(X_{\sigma(1)}\left(a_{0}\right) \delta^{a}{ }_{\sigma(2)}\left(a_{1}\right) a_{2}\right) \\
& \quad+\beta_{3} \tau\left(\delta^{a}{ }_{\sigma(1)} \delta^{b}{ }_{\sigma(2)}\left(a_{0}\right) Y_{b}\left(a_{1}\right) a_{2}\right)+\beta_{4} \tau\left(Y_{b}\left(a_{0}\right) \delta^{a}{ }_{\sigma(1)} \delta^{b}{ }_{\sigma(2)}\left(a_{1}\right) a_{2}\right) \\
& \quad+\beta_{5} \tau\left(\delta^{a}{ }_{\sigma(1)} Y_{b}\left(a_{0}\right) \delta^{b}{ }_{\sigma(2)}\left(a_{1}\right) a_{2}\right)+\beta_{6} \tau\left(\delta^{b}{ }_{\sigma(1)} Y_{b}\left(a_{0}\right) \delta^{a}{ }_{\sigma(2)}\left(a_{1}\right) a_{2}\right) \\
&+\beta_{7} \tau\left(\delta^{a}{ }_{\sigma(1)}\left(a_{0}\right) \delta^{b}{ }_{\sigma(2)} Y_{b}\left(a_{1}\right) a_{2}\right)+\beta_{8} \tau\left(\delta^{b}{ }_{\sigma(1)}\left(a_{0}\right) \delta^{a}{ }_{\sigma(2)} Y_{b}\left(a_{1}\right) a_{2}\right) \\
&+\beta_{9} \tau\left(\Delta\left(\delta^{a}{ }_{\sigma(1) \sigma(2)}\right)\left(a_{0} \otimes a_{1}\right) a_{2}\right)-r \tau\left(\delta^{a}{ }_{\sigma(1)}\left(a_{0}\right) \delta^{b}{ }_{\sigma(2)}\left(a_{1}\right) Y_{b}\left(a_{2}\right)\right) \\
& \\
&-r \tau\left(\delta^{b}{ }_{\sigma(1)}\left(a_{0}\right) \delta^{a}{ }_{\sigma(2)}\left(a_{1}\right) Y_{b}\left(a_{2}\right)\right) .
\end{aligned}
$$


By the integration by parts property, $(t \psi)_{1}=\psi_{1}$ if and only if

$$
\begin{aligned}
\beta_{1}-\beta_{2} & =0 \\
\beta_{2}-\beta_{3}+\beta_{4}-\beta_{5}+\beta_{6}+\beta_{7}-\beta_{8} & =0 \\
\beta_{1}-2 \beta_{2}+2 \beta_{4}-2 \beta_{5}-2 \beta_{6}-2 \beta_{9} & =0 \\
\beta_{2}+\beta_{6}+\beta_{7}+r & =0 \\
\beta_{2}-\beta_{3}+\beta_{5}+\beta_{6}-\beta_{8} & =0 \\
\beta_{5}+\beta_{8}+r & =0 \\
\beta_{3}+\beta_{5}+\beta_{6}-\beta_{7} & =0 \\
\beta_{3}-\beta_{4} & =0 \\
-\beta_{2}+\beta_{5}-\beta_{6}-2 \beta_{9} & =0 .
\end{aligned}
$$

Solving the systems (3.45) and (3.49) we obtain (3.47).

Finally we determine $\gamma_{k}, 1 \leq k \leq 4$ such that $\psi \in C_{\mathcal{K}}^{2}(\mathcal{A}, V)$ is a Hochschild cocycle.

Lemma 3.11. We have $(b \psi)_{0}=0$ if and only if

$$
\gamma_{1}=\gamma_{2}=r, \quad \gamma_{3}=\gamma_{4}=s .
$$

Proof. By the Koszul coaction (3.41),

$b \psi\left(1 \otimes a_{0} \otimes a_{1} \otimes a_{2} \otimes a_{3}\right)=$

$\psi_{0}\left(1 \otimes a_{0} a_{1} \otimes a_{2} \otimes a_{3}\right)-\psi_{0}\left(1 \otimes a_{0} \otimes a_{1} a_{2} \otimes a_{3}\right)$

$+\psi_{0}\left(1 \otimes a_{0} \otimes a_{1} \otimes a_{2} a_{3}\right)-\psi_{0}\left(1 \otimes a_{3} a_{0} \otimes a_{1} \otimes a_{2}\right)$

$+\psi_{1}\left(R^{a} \otimes Y_{a}\left(a_{3}\right) a_{0} \otimes a_{1} \otimes a_{2}\right)-\frac{1}{2 !} \psi_{2}\left(R^{a b} \otimes Y_{b} Y_{a}\left(a_{3}\right) a_{0} \otimes a_{1} \otimes a_{2}\right)$.

As a result, $(b \psi)_{0}=0$ if and only if

$$
\begin{gathered}
\gamma_{1}\left(-1 \otimes \delta^{a}{ }_{\sigma(1)} \otimes Y_{a} \otimes X_{\sigma(2)}+1 \otimes X_{\sigma(1)} \otimes \delta^{a}{ }_{\sigma(2)} \otimes Y_{a}\right) \\
+\gamma_{2}\left(1 \otimes \delta^{a}{ }_{\sigma(1)} \otimes X_{\sigma(2)} \otimes Y_{a}+1 \otimes \delta^{a}{ }_{\sigma(1)} \otimes Y_{a} \otimes X_{\sigma(2)}\right. \\
\left.+1 \otimes \delta^{a}{ }_{\sigma(1)} \otimes \delta^{b}{ }_{\sigma(2)} Y_{a} \otimes Y_{b}+1 \otimes \delta^{a}{ }_{\sigma(1)} \otimes \delta^{b}{ }_{\sigma(2)} \otimes Y_{b} Y_{a}\right) \\
+\gamma_{3}\left(-1 \otimes \delta^{a}{ }_{\sigma(1)} \delta^{b}{ }_{\sigma(2)} \otimes Y_{b} \otimes Y_{a}-1 \otimes Y_{b} \otimes \delta^{a}{ }_{\sigma(1)} \delta^{b}{ }_{\sigma(2)} \otimes Y_{a}\right. \\
-1 \otimes \delta^{a}{ }_{\sigma(1)} Y_{b} \otimes \delta^{b}{ }_{\sigma(2)} \otimes Y_{a}-1 \otimes \delta^{a}{ }_{\sigma(1)} \otimes \delta^{b}{ }_{\sigma(2)} Y_{b} \otimes Y_{a} \\
\left.-1 \otimes \delta^{b}{ }_{\sigma(2)} Y_{b} \otimes \delta^{a}{ }_{\sigma(1)} \otimes Y_{a}-1 \otimes \delta^{b}{ }_{\sigma(2)} \otimes \delta^{a}{ }_{\sigma(1)} Y_{b} \otimes Y_{a}\right) \\
-\gamma_{4} 1 \otimes \\
\Delta\left(\delta^{a}{ }_{\sigma(1) \sigma(2)}\right) \otimes Y_{a}-r 1 \otimes \delta^{a}{ }_{\sigma(1)} \otimes X_{\sigma(2)} \otimes Y_{a}
\end{gathered}
$$


$+-r 1 \otimes X_{\sigma(1)} \otimes \delta^{a}{ }_{\sigma(2)} \otimes Y_{a}+s 1 \otimes \delta^{a}{ }_{\sigma(1)} \delta^{b}{ }_{\sigma(2)} \otimes Y_{b} \otimes Y_{a}$

$+s 1 \otimes Y_{b} \otimes \delta^{a}{ }_{\sigma(1)} \delta^{b}{ }_{\sigma(2)} \otimes Y_{a}+s 1 \otimes \delta^{a}{ }_{\sigma(1)} Y_{b} \otimes \delta^{b}{ }_{\sigma(2)} \otimes Y_{a}$

$-s 1 \otimes \delta^{b}{ }_{\sigma(1)} Y_{b} \otimes \delta^{a}{ }_{\sigma(2)} \otimes Y_{a}+s 1 \otimes \delta^{a}{ }_{\sigma(1)} \otimes \delta^{b}{ }_{\sigma(2)} Y_{b} \otimes Y_{a}$

$+(-r-s) 1 \otimes \delta^{b}{ }_{\sigma(1)} \otimes \delta^{a}{ }_{\sigma(2)} Y_{b} \otimes Y_{a}+\left(\frac{r}{2}+s\right) 1 \otimes \Delta\left(\delta^{a}{ }_{\sigma(1) \sigma(2)}\right) \otimes Y_{a}$

$-r 1 \otimes \delta^{a}{ }_{\sigma(1)} \otimes \delta^{b}{ }_{\sigma(2)} \otimes Y_{b} Y_{a}-\frac{r}{2} 1 \otimes \Delta\left(\delta^{a}{ }_{\sigma(1) \sigma(2)}\right) \otimes Y_{a}=0$.

Hence we obtain (3.50).

Proposition 3.12. The cochain $\psi: V \otimes \mathcal{A}^{\otimes 3} \rightarrow \mathbb{C}$ is a cyclic 2-cocycle if and only if (3.44), (3.47), and (3.50) are satisfied. The resulting cocycle is then a SAYD-twisted cyclic cocycle.

Proof. We note that $\psi$ is a Hochschild cocycle, i.e. $b \psi=(b \psi)_{0}+(b \psi)_{1}+(b \psi)_{2}$ $=0$, if and only if $(b \psi)_{t}=0, t=0,1,2$. We see that $(b \psi)_{2}=0$ via Lemma 3.7, $(b \psi)_{1}=0$ via Lemma 3.9, $(b \psi)_{0}=0$ via Lemma 3.11.

On the other hand $\psi$ is cyclic, i.e. $\tau \psi=\psi$, if and only if $(\tau \psi)_{t}=\psi_{t}$, $t=0,1,2$. Indeed, for $t=1$ Lemma 3.10, for $t=2$ Lemma 3.8 yields the claims. As for $t=0$ we have

$\tau \psi\left(1 \otimes a_{0} \otimes a_{1} \otimes a_{2}\right)=\psi_{0}\left(1 \otimes a_{2} \otimes a_{0} \otimes a_{1}\right)-\psi_{1}\left(R^{a} \otimes Y_{a}\left(a_{2}\right) \otimes a_{0} \otimes a_{1}\right)$

$$
+\frac{1}{2 !} \psi_{2}\left(R^{a b} \otimes Y_{b} Y_{a}\left(a_{2}\right) \otimes a_{0} \otimes a_{1}\right) .
$$

Accordingly,

$$
\tau \psi\left(1 \otimes a_{0} \otimes a_{1} \otimes a_{2}\right)=(3.52)+(3.53)+(3.54)+(3.55)
$$

with

$$
\begin{aligned}
& r \tau(\left.X_{\sigma(1)}\left(a_{0}\right) X_{\sigma(2)}\left(a_{1}\right) a_{2}\right)+r \tau\left(\delta^{a}{ }_{\sigma(1)}\left(a_{0}\right) X_{\sigma(2)} Y_{a}\left(a_{1}\right) a_{2}\right) \\
&+s \tau\left(\delta^{a}{ }_{\sigma(1)} \delta_{\sigma(2)}^{b} Y_{b}\left(a_{0}\right) Y_{a}\left(a_{1}\right) a_{2}\right)+s \tau\left(\delta^{a}{ }_{\sigma(1) \sigma(2)}\left(a_{0}\right) Y_{a}\left(a_{1}\right) a_{2}\right), \\
& r \tau\left(\delta^{a}{ }_{\sigma(1)}\left(a_{0}\right) X_{\sigma(2)}\left(a_{1}\right) Y_{a}\left(a_{2}\right)\right)+r \tau\left(X_{\sigma(1)}\left(a_{0}\right) \delta^{a}{ }_{\sigma(2)}\left(a_{1}\right) Y_{a}\left(a_{2}\right)\right) \\
& \quad-s \tau\left(\delta^{a}{ }_{\sigma(1)} \delta_{\sigma(2)}^{b}\left(a_{0}\right) Y_{b}\left(a_{1}\right) Y_{a}\left(a_{2}\right)\right)-s \tau\left(Y_{b}\left(a_{0}\right) \delta^{a}{ }_{\sigma(1)} \delta_{\sigma(2)}^{b}\left(a_{1}\right) Y_{a}\left(a_{2}\right)\right) \\
&+(r+s) \tau\left(\delta_{\sigma(1)}^{b}\left(a_{0}\right) \delta^{a}{ }_{\sigma(2)} Y_{b}\left(a_{1}\right) Y_{a}\left(a_{2}\right)\right)-s \tau\left(\delta^{a}{ }_{\sigma(1)} Y_{b}\left(a_{0}\right) \delta_{\sigma(2)}^{b}\left(a_{1}\right) Y_{a}\left(a_{2}\right)\right) \\
&+s \tau\left(\delta_{\sigma(1)}^{b} Y_{b}\left(a_{0}\right) \delta^{a}{ }_{\sigma(2)}\left(a_{1}\right) Y_{a}\left(a_{2}\right)\right)-s \tau\left(\delta^{a}{ }_{\sigma(1)}\left(a_{0}\right) \delta_{\sigma(2)}^{b} Y_{b}\left(a_{1}\right) Y_{a}\left(a_{2}\right)\right),
\end{aligned}
$$




$$
\left(-\frac{r}{2}-s\right) \tau\left(\Delta\left(\delta_{\sigma(1) \sigma(2)}^{a}\right)\left(a_{0} \otimes a_{1}\right) Y_{a}\left(a_{2}\right)\right),
$$

and

$$
\frac{r}{2 !} \tau\left(\delta^{a}{ }_{\sigma(1)}\left(a_{0}\right) \delta_{\sigma(2)}^{b}\left(a_{1}\right) Y_{b} Y_{a}\left(a_{2}\right)\right)+\frac{r}{2 !} \tau\left(\delta_{\sigma(1)}^{b}\left(a_{0}\right) \delta^{a}{ }_{\sigma(2)}\left(a_{1}\right) Y_{b} Y_{a}\left(a_{2}\right)\right) .
$$

Using once again the integration by parts property to put the above expressions into the standard form $\tau\left(a_{0} h^{1}\left(a_{1}\right) h^{2}\left(a_{2}\right)\right)$, we obtain

$$
\tau \psi\left(1 \otimes a_{0} \otimes a_{1} \otimes a_{2}\right)=\psi_{0}\left(1 \otimes a_{0} \otimes a_{1} \otimes a_{2}\right) .
$$

We can simplify this cocycle as follows.

Theorem 3.13. The cochain $\varphi=\varphi_{0}+\varphi_{1}+\varphi_{2} \in C_{\mathcal{K}}^{2}(\mathcal{A}, V)$,

$$
\begin{gathered}
\varphi_{2}=\chi_{\tau}^{\mathrm{eq}}\left(S_{a b} \otimes \mathbf{1} \otimes \delta^{a}{ }_{\sigma(1)} \otimes \delta^{b}{ }_{\sigma(2)}+S_{a b} \otimes \mathbf{1} \otimes \delta^{b}{ }_{\sigma(1)} \otimes \delta^{a}{ }_{\sigma(2)}\right) \\
\varphi_{1}=\chi_{\tau}^{\mathrm{eq}}\left(-S_{a} \otimes \mathbf{1} \otimes \delta^{a}{ }_{\sigma(1)} \otimes X_{\sigma(2)}-S_{a} \otimes \mathbf{1} \otimes X_{\sigma(1)} \otimes \delta^{a}{ }_{\sigma(2)}\right. \\
\left.-S_{a} \otimes \mathbf{1} \otimes \delta^{b}{ }_{\sigma(1)} \otimes \delta^{a}{ }_{\sigma(2)} Y_{b}+\frac{1}{2} S_{a} \otimes \mathbf{1} \otimes \Delta\left(\delta^{a}{ }_{\sigma(1) \sigma(2)}\right)\right) \\
\varphi_{0}=\chi_{\tau}^{\mathrm{eq}}\left(1^{*} \otimes \mathbf{1} \otimes X_{\sigma(1)} \otimes X_{\sigma(2)}+1^{*} \otimes \mathbf{1} \otimes \delta^{a}{ }_{\sigma(1)} \otimes X_{\sigma(2)} Y_{a}\right),
\end{gathered}
$$

is cohomologous to $\psi$ which is defined in Proposition 3.12.

Proof. As a result of Proposition 3.12 we can write $\psi=r \varphi+s \phi$ for a 2-cochain $\phi=\phi_{0}+\phi_{1}+\phi_{2}$ given by

$$
\begin{aligned}
& \phi_{2}=0 \\
& \begin{aligned}
\phi_{1}=\chi_{\tau}^{\mathrm{eq}}( & S_{a} \otimes \mathbf{1} \otimes \delta^{a}{ }_{\sigma(1)} \delta^{b}{ }_{\sigma(2)} \otimes Y_{b}+S_{a} \otimes \mathbf{1} \otimes Y_{b} \otimes \delta^{a}{ }_{\sigma(1)} \delta^{b}{ }_{\sigma(2)} \\
& +S_{a} \otimes \mathbf{1} \otimes \delta^{a}{ }_{\sigma(1)} Y_{b} \otimes \delta^{b}{ }_{\sigma(2)}-S_{a} \otimes \mathbf{1} \otimes \delta^{b}{ }_{\sigma(1)} Y_{b} \otimes \delta^{a}{ }_{\sigma(2)} \\
& +S_{a} \otimes \mathbf{1} \otimes \delta^{a}{ }_{\sigma(1)} \otimes \delta^{b}{ }_{\sigma(2)} Y_{b}-S_{a} \otimes \mathbf{1} \otimes \delta^{b}{ }_{\sigma(1)} \otimes \delta^{a}{ }_{\sigma(2)} Y_{b} \\
& \left.+S_{a} \otimes \mathbf{1} \otimes \Delta\left(\delta^{a}{ }_{\sigma(1) \sigma(2)}\right)\right)
\end{aligned} \\
& \phi_{0}=\chi_{\tau}^{\mathrm{eq}}\left(1^{*} \otimes \mathbf{1} \otimes \delta^{a}{ }_{\sigma(1)} \delta^{b}{ }_{\sigma(2)} Y_{b} \otimes Y_{a}+1^{*} \otimes \mathbf{1} \otimes \delta^{a}{ }_{\sigma(1) \sigma(2)} \otimes Y_{a}\right)
\end{aligned}
$$

We note that

$$
\phi_{1}=\chi_{\tau}^{\mathrm{eq}}\left(S_{a} \otimes \mathbf{1} \otimes \Delta\left(\delta^{a}{ }_{\sigma(1)} \delta^{b}{ }_{\sigma(2)} Y_{b}\right)+S_{a} \otimes \mathbf{1} \otimes \Delta\left(\delta^{a}{ }_{\sigma(1) \sigma(2)}\right)\right) .
$$

It is then straightforward to check that the 1-cochain $\phi^{\prime}=\phi_{0}^{\prime}+\phi_{1}^{\prime}+\phi_{2}^{\prime}$ given by

$$
\phi_{2}^{\prime}=0
$$




$$
\begin{aligned}
\phi_{1}^{\prime} & =\chi_{\tau}^{\mathrm{eq}}\left(S_{a} \otimes \mathbf{1} \otimes \delta^{a}{ }_{\sigma(1)} \delta^{b}{ }_{\sigma(2)} Y_{b}+S_{a} \otimes \mathbf{1} \otimes \delta^{a}{ }_{\sigma(1) \sigma(2)}\right) \\
\phi_{0}^{\prime} & =0
\end{aligned}
$$

is an equivariant cyclic 1-cocycle, and that

$$
b \phi^{\prime}=\phi .
$$

\section{The characteristic map with coefficients}

In this section the main object of the paper, which is a characteristic map from the truncated Weil complex of the Lie algebra $\mathfrak{g}_{0}$ to the cyclic complex of the algebra $\mathcal{A}$, is constructed. We illustrate the characteristic map in codimensions $n=1$ and $n=2$ and observe that the resulting cocycles in codimension 1 match with those in $[2,3]$ by Connes-Moscovici .

Such a characteristic map is obtained by composing a series of maps

$$
H\left(W\left(\mathfrak{g}_{0}, V\right)\right) \stackrel{\mathfrak{D}_{P}}{\longrightarrow} H\left(C\left(\mathfrak{g}_{0}, V\right)\right) \stackrel{\cong}{\longrightarrow} H C(\mathcal{K}, V) \stackrel{\chi_{\varphi}}{\longrightarrow} H C(\mathcal{A}) .
$$

As it is shown in [13] the truncated Weil algebra is identical with $W\left(\mathfrak{g}_{0}, V\right)$. The Poincare isomorphism $\mathfrak{D}_{\mathrm{P}}$ is defined in [13, Prop. 4.4]. The middle quasiisomorphism is defined in [13, Thm. 6.2]. Finally the map $\chi_{\varphi}$ is given by the cup product, in the sense of $[8,12]$, with the SAYD-twisted cyclic cocycle $\varphi$ defined in Proposition 3.6 for codimension 1, in Theorem 3.13 for codimension 2.

Let us recall the above mentioned cup product from [12]. Let $C$ be a $H$-module coalgebra and $A$ be an $H$-module algebra that are equipped with a mapping

$$
C \otimes A \rightarrow A, \quad c \otimes a \mapsto c(a)
$$

satisfying the conditions

$$
(h \cdot c)(a)=h \cdot(c(a)), \quad c(a b)=c_{(1)}(a) c_{(2)}(b), \quad c(1)=\varepsilon(c) 1 .
$$

Let also $V$ be a SAYD module over a Hopf algebra $H$. One defines

$$
\cup: C_{H}^{p}(C, V) \otimes C_{H}^{q}(A, V) \rightarrow C^{p+q}(A)
$$

for any $\varphi \in C_{H}^{q}(A, V)$ and any $x=v \otimes_{H} c^{0} \otimes \cdots \otimes c^{p} \in C_{H}^{p}(C, V)$,

$$
\begin{aligned}
& (x \cup \varphi)\left(a_{0} \otimes \cdots \otimes a_{p+q}\right):= \\
& \sum_{\sigma \in \operatorname{Sh}(p, q)}(-1)^{\sigma} \partial_{\bar{\sigma}(p)} \ldots \partial_{\bar{\sigma}(1)} \varphi\left(\left\langle\partial_{\bar{\sigma}(p+q)} \ldots \partial_{\bar{\sigma}(p+1)} x, a_{0} \otimes \cdots \otimes a_{p+q}\right\rangle\right),
\end{aligned}
$$

where $\left\langle x, a_{0} \otimes \cdots \otimes a_{n}\right\rangle:=v \otimes_{H} c^{0}\left(a^{0}\right) \otimes \cdots \otimes c^{n}\left(a^{n}\right), \operatorname{Sh}(p, q)$ is the set of all $(p, q)$-shuffle permutations, and $\bar{\sigma}(n)=\sigma(n)-1$. 
We set $C=H:=\mathcal{K}, \quad V=S\left(\mathfrak{g}_{0}^{*}\right)_{[2 n]}, \quad A=\mathcal{A}$. Then $H$ acts on $C$ via multiplication, on $A$ as (3.22), and on $V$ via the coadjoint action. The construction yields for any $\varphi \in C_{\mathcal{K}}^{n}(\mathcal{A}, V)$ a characteristic map

$$
\chi_{\varphi}: C_{\mathcal{K}}^{\bullet}(\mathcal{K}, V) \rightarrow C^{\bullet+n}(\mathcal{A}) .
$$

4.1. The characteristic map in codimension 1. In this subsection we use the SAYD-twisted cyclic cocycle of Proposition 3.6 to illustrate (4.1) in codimension $n=1$.

In order to verify that the new characteristic map is geometrically meaningful, we compare its image with the transverse fundamental class $T F:=\chi_{\tau}(\mathrm{TF})=$ $\chi_{\tau}\left(X \otimes Y-Y \otimes X-\delta_{1} Y \otimes Y\right) \in H C^{2}(\mathcal{A})$,

$$
\begin{aligned}
T F\left(a_{0} \otimes\right. & \left.a_{1} \otimes a_{2}\right) \\
& =\tau\left(a_{0} X\left(a_{1}\right) Y\left(a_{2}\right)\right)-\tau\left(a_{0} Y\left(a_{1}\right) X\left(a_{2}\right)\right)-\tau\left(a_{0} \delta_{1} Y\left(a_{1}\right) Y\left(a_{2}\right)\right)
\end{aligned}
$$

and the Godbillon-Vey class $G V:=\chi_{\tau}(\mathrm{GV})=\chi_{\tau}\left(\delta_{1}\right) \in H C^{1}(\mathcal{A})$,

$$
G V\left(a_{0} \otimes a_{1}\right)=\tau\left(a_{0} \delta_{1}\left(a_{1}\right)\right) .
$$

The next step is to find the representative cocycles in $H\left(W\left(\mathfrak{g}_{0}, V\right), d\right)$ of (2.27). Let $\{Y\}$ and $\{\theta\}$ be a dual pair of bases for $\mathfrak{g}_{0}$ and $\mathfrak{g}_{0}^{*}$.

By the Vey basis [4], the cohomology of $W\left(\mathfrak{g}_{0}\right)_{[2]}$ is spanned by

$$
\mathrm{TF}:=1 \in S\left(\mathfrak{g}_{0}^{*}\right)_{[2]}, \quad \mathrm{GV}:=\theta \otimes R \in \mathfrak{g}_{0}^{*} \otimes S\left(\mathfrak{g}_{0}^{*}\right)_{[2]}
$$

Applying the Poincaré duality [13, Prop. 4.4], we obtain

$$
\mathfrak{D}_{\mathrm{P}}(1)=Y \otimes 1 \in \mathfrak{g}_{0} \otimes V, \quad \mathfrak{D}_{\mathrm{P}}(\theta \otimes R)=R \in V .
$$

Proposition 4.1. The Hopf-cyclic cohomology $H C(\mathcal{K}, V)$ is generated by the classes

$$
\begin{aligned}
{[R] } & \in H C^{0}(\mathcal{K}, V), \\
{\left[1 \otimes Y+\frac{1}{2} R \otimes Y^{2}\right] } & \in H C^{1}(\mathcal{K}, V) .
\end{aligned}
$$

Proof. It is straightforward to check that $R \in C^{0}(\mathcal{K}, V)$ and $1 \otimes Y+\frac{1}{2} R \otimes Y^{2} \in$ $C^{1}(\mathcal{K}, V)$ are cyclic cocycles. The claim, then follows from the observation

$$
\mu(R)=R, \quad \mu\left(1 \otimes Y+\frac{1}{2} R \otimes Y^{2}\right)=1 \otimes Y,
$$

for the quasi-isomorphism

$$
\mu: C^{\bullet}(U(\mathfrak{g}), V) \longrightarrow C_{\bullet}(\mathfrak{g}, V),
$$

which, for any Lie algebra $\mathfrak{g}$, is the left inverse of the anti-symmetrization map, see $[3,13]$. 
Next, we compute

$$
\begin{aligned}
\chi_{\varphi}(\theta)\left(a_{0} \otimes a_{1}\right) & =(\varphi \cup(\theta \otimes 1))\left(a_{0} \otimes a_{1}\right)=\varphi\left(\left\langle\partial_{0}(\theta \otimes 1), a_{0} \otimes a_{1}\right\rangle\right) \\
& =\varphi\left(\theta \otimes a_{0} \otimes a_{1}\right)=-\tau\left(a_{0} \delta_{1}\left(a_{1}\right)\right),
\end{aligned}
$$

and in the same way,

$$
\begin{aligned}
\chi_{\varphi}\left(1 \otimes Y+\frac{1}{2} \theta \otimes Y^{2}\right)\left(a_{0} \otimes a_{1} \otimes a_{2}\right) \\
=\sum_{\sigma \in S h(1,1)}(-1)^{\sigma} \partial_{\bar{\sigma}(1)} \varphi\left(\left\langle\partial_{\bar{\sigma}(2)}\left(1 \otimes Y+\frac{1}{2} \theta \otimes Y^{2}\right), a_{0} \otimes a_{1} \otimes a_{2}\right\rangle\right) \\
=-\partial_{0} \varphi\left(\left\langle\partial_{1}\left(1 \otimes Y+\frac{1}{2} \theta \otimes Y^{2}\right), a_{0} \otimes a_{1} \otimes a_{2}\right\rangle\right) \\
\quad+\partial_{1} \varphi\left(\left\langle\partial_{0}\left(1 \otimes Y+\frac{1}{2} \theta \otimes Y^{2}\right), a_{0} \otimes a_{1} \otimes a_{2}\right\rangle\right) \\
=\varphi\left(-1 \otimes a_{0} a_{1} \otimes Y\left(a_{2}\right)-1 \otimes a_{0} Y\left(a_{1}\right) \otimes a_{2}-\frac{1}{2} \theta \otimes a_{0} Y^{2}\left(a_{1}\right) \otimes a_{2}\right. \\
\left.\quad-\frac{1}{2} \theta \otimes a_{0} a_{1} \otimes Y^{2}\left(a_{2}\right)-\theta \otimes a_{0} Y\left(a_{1}\right) \otimes Y\left(a_{2}\right)\right) \\
\quad+\varphi\left(1 \otimes a_{0} \otimes a_{1} Y\left(a_{2}\right)+\frac{1}{2} \theta \otimes a_{0} \otimes a_{1} Y^{2}\left(a_{2}\right)\right) \\
\left.\left.\quad+\frac{1}{2} \tau\left(a_{0} \delta_{1}\left(a_{1}\right) Y^{2}\left(a_{2}\right)\right)+\tau\left(a_{1}\right) X\left(a_{2}\right)\right)+\tau\left(a_{0} X\left(a_{1}\right) Y\left(a_{2}\right)\right)+\frac{1}{2} \tau\left(a_{0} Y^{2}\left(a_{1}\right) \delta_{1}\left(a_{2}\right)\right)\right)
\end{aligned}
$$

As a result,

$$
\chi_{\varphi}\left(1 \otimes Y+\frac{1}{2} \theta \otimes Y^{2}\right)=\chi_{\tau}\left(\mathrm{TF}+\frac{1}{2} b\left(\delta_{1} Y^{2}\right)\right)
$$

that is, we obtain the transverse fundamental class up to a coboundary. Similarly we obtain the Godbillon-Vey class

$$
\chi_{\varphi}(\theta)=-\chi_{\tau}(\mathrm{GV})
$$

4.2. The characteristic map in codimension 2 . In this subsection we demonstrate the machinery we developed in Subsection 3.3 for codimension $n=2$. We note that there is no such computations in the literature that we know of.

Let us fix the following notation

$$
\begin{gathered}
c_{1}=\operatorname{Tr}=R_{1}^{1}+R_{2}^{2} \in S\left(\mathfrak{g}_{0}^{*}\right), \quad c_{2}=R_{2}^{1} R_{1}^{2} \in S\left(\mathfrak{g}_{0}^{*}\right), \\
u_{1}=\theta_{1}^{1}+\theta_{2}^{2}, \quad u_{2}=\theta_{1}^{1} \wedge \theta_{2}^{1} \wedge \theta_{1}^{2}, \quad \omega=\theta_{1}^{1} \wedge \theta_{2}^{1} \wedge \theta_{1}^{2} \wedge \theta_{2}^{2} .
\end{gathered}
$$

The Vey basis, [4], for $W\left(\mathfrak{g}_{0}\right)_{[4]}$ is then introduced by

$$
\left\{1, c_{1}^{2} \otimes u_{1}, c_{2} \otimes u_{1}, c_{2} \otimes u_{2}, c_{1}^{2} \otimes \omega, c_{2} \otimes \omega\right\} .
$$


Next, the Poincaré duality yields the 6 cocycles in the complex $C\left(\mathfrak{g}_{0}, V\right)$ :

$$
\begin{aligned}
\mathfrak{D}_{\mathrm{P}}(1) & =1 \otimes Y_{1}^{1} \wedge Y_{1}^{2} \wedge Y_{2}^{1} \wedge Y_{2}^{2}, \\
\mathfrak{D}_{\mathrm{P}}\left(c_{2} \otimes u_{1}\right) & =c_{2} \otimes\left(Y_{1}^{2} \wedge Y_{2}^{1} \wedge Y_{2}^{2}-Y_{1}^{1} \wedge Y_{1}^{2} \wedge Y_{2}^{1}\right), \\
\mathfrak{D}_{\mathrm{P}}\left(c_{1}^{2} \otimes u_{1}\right) & =c_{1}^{2} \otimes\left(Y_{1}^{2} \wedge Y_{2}^{1} \wedge Y_{2}^{2}-Y_{1}^{1} \wedge Y_{1}^{2} \wedge Y_{2}^{1}\right), \\
\mathfrak{D}_{\mathrm{P}}\left(c_{2} \otimes u_{2}\right) & =c_{2} \otimes Y_{2}^{2}, \\
\mathfrak{D}_{\mathrm{P}}\left(c_{1}^{2} \otimes \omega\right) & =c_{1}^{2}, \\
\mathfrak{D}_{\mathrm{P}}\left(c_{2} \otimes \omega\right) & =c_{2} .
\end{aligned}
$$

Let us label $Y_{i}^{j}$ as $Y_{1}:=Y_{1}^{1}, \quad Y_{2}:=Y_{1}^{2}, \quad Y_{3}:=Y_{2}^{1}, \quad Y_{4}:=Y_{2}^{2}$.

Proposition 4.2. The Hopf-cyclic cohomology $H C(\mathcal{K}, V)$ is generated by the classes

$$
\begin{aligned}
& {[\mathscr{T} \mathscr{F}] } \in H C^{4}(\mathcal{K}, V) \\
& {[\mathscr{G} \mathscr{V}] }:=\left[\sum _ { \sigma \in S _ { 3 } } ( - 1 ) ^ { \sigma } c _ { 1 } ^ { 2 } \otimes \left(Y_{\sigma(2)} \otimes Y_{\sigma(3)} \otimes Y_{\sigma(4)}\right.\right. \\
&\left.\left.-Y_{\sigma(1)} \otimes Y_{\sigma(2)} \otimes Y_{\sigma(3)}\right)\right] \in H C^{3}(\mathcal{K}, V), \\
& {\left[\mathscr{R}_{1}\right] }:=\left[\sum _ { \sigma \in S _ { 3 } } ( - 1 ) ^ { \sigma } c _ { 2 } \otimes \left(Y_{\sigma(2)} \otimes Y_{\sigma(3)} \otimes Y_{\sigma(4)}\right.\right. \\
&\left.\left.\quad-Y_{\sigma(1)} \otimes Y_{\sigma(2)} \otimes Y_{\sigma(3)}\right)\right] \in H C^{3}(\mathcal{K}, V), \\
& {\left[\mathscr{R}_{2}\right]:=\left[c_{2} \otimes Y_{4}\right] \in H C^{1}(\mathcal{K}, V), } \\
& {\left[\mathscr{R}_{3}\right]:=\left[c_{1}^{2}\right] \in H C^{0}(\mathcal{K}, V), } \\
& {\left[\mathscr{R}_{4}\right]:=\left[c_{2}\right] \in H C^{0}(\mathcal{K}, V) . }
\end{aligned}
$$

Proof. It is straightforward to check that $\mathscr{R}_{1}, \ldots, \mathscr{R}_{4}$ and $\mathscr{G} \mathscr{V}$ are Hopf-cyclic cocycles and that

$$
\begin{aligned}
\mu\left(\mathscr{R}_{1}\right) & =\mathfrak{D}_{\mathrm{P}}\left(c_{2} \otimes u_{1}\right), & & \mu\left(\mathscr{R}_{2}\right)=\mathfrak{D}_{\mathrm{P}}\left(c_{2} \otimes u_{2}\right), \\
\mu\left(\mathscr{R}_{3}\right) & =\mathfrak{D}_{\mathrm{P}}\left(c_{1}^{2} \otimes \omega\right), & & \mu\left(\mathscr{R}_{4}\right)=\mathfrak{D}_{\mathrm{P}}\left(c_{2} \otimes \omega\right), \\
\mu(\mathscr{G} \mathscr{V}) & =\mathfrak{D}_{\mathrm{P}}\left(c_{1}^{2} \otimes u_{1}\right) . & &
\end{aligned}
$$

On the other hand,

$$
[\mathscr{T} \mathscr{F}]=\left[\sum_{\sigma \in S_{4}}(-1)^{\sigma} 1 \otimes Y_{\sigma(1)} \otimes Y_{\sigma(2)} \otimes Y_{\sigma(3)} \otimes Y_{\sigma(4)}\right] \in E_{1}^{2,2}(\mathcal{K}, V)
$$

is a cyclic cocycle in the $E_{1}$ level of the spectral sequence that corresponds to the natural filtration of $V,[13$, Thm. 6.2]. Hence

$$
\mu(\mathscr{T} \mathscr{F})=\mathfrak{D}_{\mathrm{P}}(1) .
$$

Therefore, the claim follows from [13, Thm. 6.2]. 
In this paper we do not complete the fundamental cocycle as we know its counterpart as a cyclic cocycle over $\mathcal{A}$ by the following argument. Let us recall the characteristic map

$$
\chi_{\varphi}: C^{\bullet}(\mathcal{K}, V) \longrightarrow C^{\bullet+2}(\mathcal{A})
$$

for the SAYD-twisted cyclic 2-cocycle defined by the Theorem 3.13. To this end, we first prove a generalization of [3, Prop. 18]. In view of [10], $\mathcal{H}:=\mathcal{H}_{n}$ is realized as a bicrossed product Hopf algebra $\mathcal{U} \bowtie \mathcal{F}^{\text {cop }}$. Here $\mathcal{F}$ is the commutative algebra of regular functions on the group of diffeomorphisms which preserve the origin and with identity Jacobian at the origin, and $\mathcal{U}=U\left(g \ell_{2}^{\text {affine }}\right)$. The coaction involed in this bicrossed product realization is recalled below

$$
\nabla: \mathcal{U} \rightarrow \mathcal{F}^{\text {cop }} \otimes \mathcal{U}, \quad X_{k} \mapsto 1 \otimes X_{k}+\delta_{j k}^{i} \otimes Y_{i}^{j}, \quad Y_{i}^{j} \mapsto 1 \otimes Y_{i}^{j} .
$$

In the following proposition, for any $1 \leq j \leq m:=n^{2}+n$,

$$
\nabla^{j}(Z)=Z_{<-j>} \otimes \cdots \otimes Z_{<-1>} \otimes Z_{<0>} \otimes 1 \otimes \cdots \otimes 1 \in \mathcal{H}^{\otimes m+1} .
$$

Proposition 4.3. The $m:=n^{2}+n$-cochain

$$
\mathrm{TF}:=(-1)^{(m-1) !} \sum_{\sigma \in S_{m}}(-1)^{\sigma} \nabla^{m}\left(Z^{\sigma(1)}\right) \cdots \nabla\left(Z^{\sigma(m)}\right) \in \mathcal{H}^{\otimes m+1}
$$

is a cyclic m-cocycle whose class $[\mathrm{TF}] \in H C^{m}(\mathcal{H})$ corresponds, by the ConnesMoscovici characteristic map, to the transverse fundamental class $[T F] \in H C^{m}(\mathcal{A})$.

Proof. Let $a^{i}:=f^{i} U_{\psi_{i}}^{*} \in \mathcal{A}$, where $0 \leq i \leq m, f^{i} \in C_{c}^{\infty}\left(F^{+}\right)$and $\psi \in \Gamma$. Without loss of generality we assume that $\psi_{m} \ldots \psi_{0}=$ Id. The cyclic cocycle $T F \in H C^{m}(\mathcal{A})$ is given by the $m$-cocycle

$$
T F\left(a^{0} \otimes \cdots \otimes a^{m}\right)=\int_{F^{+}} a^{0} d a^{1} \cdots d a^{m}, \quad d a^{i}=d f^{i} U_{\psi_{i}}^{*} .
$$

In order to prove the claim, we need to find $h^{0}, \ldots, h^{m} \in \mathcal{H}$ such that

$$
T F\left(a^{0} \otimes \cdots \otimes a^{m}\right)=\tau\left(h^{0}\left(a^{0}\right) \cdots h^{m}\left(a^{m}\right)\right) .
$$

Indeed,

$$
\begin{aligned}
& \int_{F+\mathbb{R}^{n}} a^{0} d a^{1} \cdots d a^{m}=\int_{F+\mathbb{R}^{n}} f^{0} \psi_{0}{ }^{*}\left(d f^{1}\right) \cdots\left(\psi_{0}{ }^{*} \ldots \psi_{m-1}{ }^{*}\right)\left(d f^{m}\right) \\
& \quad=\int_{F+\mathbb{R}^{n}} h^{0}\left(f^{0}\right) \psi_{0}{ }^{*}\left(h^{1}\left(f^{1}\right)\right) \cdots\left(\psi_{0}{ }^{*} \ldots \psi_{m-1}{ }^{*}\right)\left(h^{m}\left(f^{m}\right)\right) \varpi \\
& \quad=\int_{F+\mathbb{R}^{n}}\left(\operatorname{Id} \otimes \psi_{0}{ }^{*} \otimes \cdots \otimes \psi_{0}{ }^{*} \ldots \psi_{m-1}{ }^{*}\right)\left(h^{0} \otimes \cdots \otimes h^{m}\right)\left(f^{0} \otimes \cdots \otimes f^{m}\right) \varpi,
\end{aligned}
$$


where the volume form on the frame bundle is

$$
\varpi=\bigwedge_{i=1}^{n} \theta^{i} \wedge \bigwedge_{1 \leq i, j \leq n} \omega_{j}^{i} \quad \text { (ordered lexicographically) }
$$

In the above computation we use the notations

$$
\left(h^{0} \otimes \cdots \otimes h^{m}\right)\left(f^{0} \otimes \cdots \otimes f^{m}\right)=h^{0}\left(f^{0}\right) \ldots h^{m}\left(f^{m}\right),
$$

and similarly for any $g^{0}, \ldots, g^{m} \in C_{c}^{\infty}\left(F^{+}\right)$,

$$
\begin{aligned}
\left(\mathrm{Id} \otimes \psi_{0}{ }^{*} \otimes \cdots \otimes \psi_{0}{ }^{*} \ldots \psi_{m-1}{ }^{*}\right)\left(g^{0} \otimes\right. & \left.\cdots \otimes g^{m}\right) \\
& =g^{0} \psi_{0}{ }^{*}\left(g^{1}\right) \ldots \psi_{0}{ }^{*} \ldots \psi_{m-1}{ }^{*}\left(g^{m}\right) .
\end{aligned}
$$

Here $\psi^{*}(g)(x, y)=g\left(\psi(x), \psi^{\prime}(x) \cdot y\right)$.

For any $f \in C_{c}^{\infty}\left(F^{+}\right)$we have

$$
d f=\frac{\partial f}{\partial x^{i}} d x^{i}+\frac{\partial f}{\partial y_{i}^{j}} d y_{i}^{j}=X_{i}(f) \theta^{i}+Y_{i}^{j}(f) \omega_{j}^{i} .
$$

Therefore, for $\psi_{0} \in \operatorname{Diff}\left(\mathbb{R}^{n}\right)$, and $f^{0}, f^{1} \in C_{c}^{\infty}\left(F^{+}\right)$we have

$$
\begin{aligned}
& f^{0} \psi_{0}{ }^{*}\left(d f^{1}\right)= f^{0} \psi_{0}{ }^{*}\left(X_{i}\left(f^{1}\right)\right) \psi_{0}{ }^{*}\left(\theta^{i}\right)+f^{0} \psi_{0}{ }^{*}\left(Y_{i}^{j}\left(f^{1}\right)\right) \psi_{0}{ }^{*}\left(\omega_{j}^{i}\right) \\
&= f^{0}{\psi_{0}}^{*}\left(X_{i}\left(f^{1}\right)\right) \theta^{i}+f^{0} \psi_{0}{ }^{*}\left(Y_{i}^{j}\left(f^{1}\right)\right)\left(\gamma_{j k}^{i}\left(\psi_{0}\right) \theta^{k}+\omega_{j}^{i}\right) \\
&=\left(\operatorname{Id} \otimes \psi_{0}{ }^{*}\right)\left[\left(1 \otimes X_{k}+\delta_{j k}^{i} \otimes Y_{i}^{j}\right)\left(f^{0} \otimes f^{1}\right) \theta^{k}\right.\left.+\left(1 \otimes Y_{i}^{j}\right)\left(f^{0} \otimes f^{1}\right) \omega_{j}^{i}\right] \\
&=\left(\operatorname{Id} \otimes \psi_{0}{ }^{*}\right)\left(\left(X_{k}\right)_{<-1>} \otimes\left(X_{k}\right)_{<0>}\right)\left(f^{0} \otimes f^{1}\right) \theta^{k} \\
& \quad+\left(\operatorname{Id} \otimes \psi_{0}{ }^{*}\right)\left(\left(Y_{i}^{j}\right)_{<-1>} \otimes\left(Y_{i}^{j}\right)_{<0>}\right)\left(f^{0} \otimes f^{1}\right) \omega_{j}^{i} .
\end{aligned}
$$

On the second equality we used [3, (2.16)], and on the third equality we used (3.22). On the forth equality, the left coaction is (4.21). 
On the other hand we have

$$
\begin{gathered}
(-1)^{(m-1) !} f^{0} \psi_{0}{ }^{*}\left(d f^{1}\right) \psi_{0}{ }^{*} \psi_{1}{ }^{*}\left(d f^{2}\right) \ldots \psi_{0}{ }^{*} \cdots \psi_{m-1}{ }^{*}\left(d f^{m}\right) \\
=\psi_{0}{ }^{*} \cdots \psi_{m-1}{ }^{*}\left(d f^{m}\right) \ldots \psi_{0}{ }^{*} \psi_{1}{ }^{*}\left(d f^{2}\right) \psi_{0}{ }^{*}\left(d f^{1}\right) f^{0} \\
=\left(\psi_{m-1} \cdots \psi_{0}{ }^{*}\left(X_{i}\left(f^{m}\right)\right) \theta^{i}+\psi_{m-1} \cdots \psi_{0}{ }^{*}\left(Y_{i}^{j}\left(f^{m}\right)\right)\right. \\
\left.\left(\gamma_{j k}^{i}\left(\psi_{m-1} \cdots \psi_{1}\right) \theta^{k}+\omega_{j}^{i}\right)\right) \cdots \\
\ldots\left(\psi_{1} \psi_{0}{ }^{*}\left(X_{i}\left(f^{2}\right)\right) \theta^{i}+\psi_{1} \psi_{0}{ }^{*}\left(Y_{i}^{j}\left(f^{2}\right)\right)\left(\gamma_{j k}^{i}\left(\psi_{2} \psi_{1}\right) \theta^{k}+\omega_{j}^{i}\right)\right) \\
\cdot\left(\psi_{0}{ }^{*}\left(X_{i}\left(f^{1}\right)\right) \theta^{i}+\psi_{0}{ }^{*}\left(Y_{i}^{j}\left(f^{1}\right)\right)\left(\gamma_{j k}^{i}\left(\psi_{1}\right) \theta^{k}+\omega_{j}^{i}\right)\right) \cdot f^{0} \\
=\left(\mathrm{Id} \otimes \psi_{0}{ }^{*} \otimes \cdots \otimes \psi_{0}{ }^{*} \cdots \psi_{m-1}{ }^{*}\right)\left\{\left[\left(\left(X_{i}\right)_{<-m>} \otimes \cdots \otimes\left(X_{i}\right)_{<0>}\right) \theta^{i}\right.\right. \\
\left.+\left(\left(Y_{i}^{j}\right)_{<-m>} \otimes \cdots \otimes\left(Y_{i}^{j}\right)_{<0>}\right) \omega_{j}^{i}\right] \cdots \\
\cdots \cdot\left[\left(\left(X_{i}\right)_{<-2>} \otimes\left(X_{i}\right)_{<-1>} \otimes\left(X_{i}\right)_{<0>} \otimes 1 \otimes \cdots \otimes 1\right) \theta^{i}\right. \\
\left.+\left(\left(Y_{i}^{j}\right)_{<-2>} \otimes\left(Y_{i}^{j}\right)_{<-1>} \otimes\left(Y_{i}^{j}\right)_{<0>} \otimes 1 \otimes \cdots \otimes 1\right) \omega_{j}^{i}\right] \\
\cdot\left[\left(\left(X_{i}\right)_{<-1>} \otimes\left(X_{i}\right)_{<0>} \otimes 1 \otimes \cdots \otimes 1\right) \theta^{i}\right. \\
\left.\left.+\left(\left(Y_{i}^{j}\right)_{<-1>} \otimes\left(Y_{i}^{j}\right)_{<0>} \otimes 1 \otimes \cdots \otimes 1\right) \omega_{j}^{i}\right]\right\}\left(f^{0} \otimes \cdots \otimes f^{m}\right) .
\end{gathered}
$$

On the third equality, we used the cocycle identity [10, (1.16)] in order to obtain the expressions in $\mathcal{H}^{\otimes m+1}$ in the range of the coaction (4.21). We reversed the order of the multiplication in (4.28) in order to avoid obtaining elements in $\mathcal{H}^{\otimes m+1}$ involving $Y_{i}^{j} \delta_{q r}^{p} \in \mathcal{H}$ which do not belong the PBW basis of $\mathcal{H}$, [3, Prop. 3].

The coefficient of the volume form (4.26), which is an element $H \in \mathcal{H}^{\otimes m+1}$, can now be expressed by carrying out the multiplication in (4.28). Let $\left(Z^{1}, \ldots, Z^{m}\right)=$ $\left(X_{1}, \ldots, X_{n}, Y_{1}^{1}, \ldots, Y_{n}^{n}\right)$, where the right hand side is ordered lexicographically. Then

$$
H=\sum_{\sigma \in S_{m}}(-1)^{\sigma} \nabla^{m}\left(Z^{\sigma(1)}\right) \cdots \nabla\left(Z^{\sigma(m)}\right) .
$$

Finally, as a result of (4.25), (4.28) and (4.29), we have the element

$$
\mathrm{TF}:=(-1)^{(m-1) !} \sum_{\sigma \in S_{m}}(-1)^{\sigma} \nabla^{m}\left(Z^{\sigma(1)}\right) \cdots \nabla\left(Z^{\sigma(m)}\right) \in \mathcal{H}^{\otimes m+1}
$$

such that $\chi_{\tau}(\mathrm{TF})=T F \in C^{m}(\mathcal{A})$. 
Let us illustrate the proposition for $n=1$. We have $\left(Z^{1}, Z^{2}\right)=(X, Y), m=$ $1^{2}+1=2$ and

$$
\begin{aligned}
\mathrm{TF}=(-1)^{1 !} \sum_{\sigma \in S_{2}}(-1)^{\sigma} \nabla^{2}\left(Z^{\sigma(1)}\right) \nabla\left(Z^{\sigma(2)}\right) & \\
=-\left(\left(X_{<-2>} \otimes X_{<-1>} \otimes\right.\right. & \left.X_{<0>}\right)\left(Y_{<-1>} \otimes Y_{<0>} \otimes 1\right) \\
& \left.\quad-\left(Y_{<-2>} \otimes Y_{<-1>} \otimes Y_{<0>}\right)\left(X_{<-1>} \otimes X_{<0>} \otimes 1\right)\right)
\end{aligned}
$$$$
=-1 \otimes Y \otimes X-1 \otimes \delta_{1} Y \otimes Y-\delta_{1} \otimes Y \otimes Y+1 \otimes X \otimes Y+\delta_{1} \otimes Y \otimes Y
$$$$
=1 \otimes X \otimes Y-1 \otimes Y \otimes X-1 \otimes \delta_{1} Y \otimes Y .
$$

Next we recall the isomorphism

$$
\begin{aligned}
& \Psi_{\bowtie}: C^{n}\left(\mathcal{H}, \mathbb{C}_{\delta}\right) \rightarrow D^{n}\left(\mathcal{U}, \mathcal{F}, \mathbb{C}_{\delta}\right) \\
& \Psi_{\bowtie}\left(u^{1} \bowtie f^{1} \otimes \ldots \otimes u^{n} \bowtie f^{n}\right)= \\
& \quad u_{<-n>}^{1} f^{1} \otimes \cdots \otimes u_{<-1>}^{1} \ldots u_{<-1>}^{n} f^{n} \otimes u_{<0>}^{1} \otimes \cdots \otimes u_{<0>}^{n}
\end{aligned}
$$

defined in [10] that identifies the Hopf-cyclic complex $C\left(\mathcal{H}, \mathbb{C}_{\delta}\right)$ of the Hopf algebra $\mathcal{H}=\mathcal{U} \bowtie \mathcal{F}$ with the diagonal subcomplex

$$
D^{\bullet}\left(\mathcal{U}, \mathcal{F}, \mathbb{C}_{\delta}\right):=\mathbb{C}_{\delta} \otimes \mathcal{F}^{\otimes \bullet} \otimes \mathcal{U}^{\otimes \bullet} .
$$

Remark 4.4. The transverse fundamental class $[\mathrm{TF}] \in H C^{n^{2}+n}\left(\mathcal{H}_{n}, \mathbb{C}_{\delta}\right)$ defined in (4.23) corresponds to the class

$$
\left[1 \otimes X_{1} \wedge \cdots \wedge X_{n} \wedge Y_{1}^{1} \wedge \cdots \wedge Y_{n}^{n}\right]
$$

in the total complex $C^{\bullet \bullet \bullet}\left(\mathfrak{g}, \mathcal{F}, \mathbb{C}_{\delta}\right)[10$, (3.37)], by the composition of (4.31) and (4.14).

On the next move, we introduce the commutative diagram

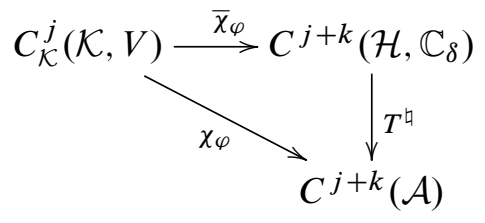

induced by (a decomposition of) the cup product (4.5) via a cyclic cocycle $\varphi \in$ $C_{\mathcal{K}}^{k}(\mathcal{A}, V)$ in the image of (3.30). Here $T^{\natural}: \mathcal{H}^{\otimes j} \rightarrow C^{j}(\mathcal{A})$ is the isomorphism defined in $[3,(3.12)]$.

We are now ready to prove our claim. On the following proposition, $\varphi \in$ $C_{\mathcal{K}}^{2}(\mathcal{A}, V)$ is the cyclic cocycle defined in Theorem 3.13. 
Proposition 4.5. The cyclic cohomology class $[\mathscr{T} \mathscr{F}] \in H C^{4}(\mathcal{K}, V)$ is mapped by $\chi_{\varphi}: H C^{4}(\mathcal{K}, V) \rightarrow H C^{6}(\mathcal{A})$ to the transverse characteristic class $[T F] \in H C^{6}(\mathcal{A})$.

Proof. By the diagram (4.34) we understand that it is enough to observe $\left[\bar{\chi}_{\varphi}(\mathscr{T} \mathscr{F})\right]=$ $[\mathrm{TF}] \in H C^{6}(\mathcal{H})$. This, in turn, follows from

$$
\mu \circ \psi_{\bowtie}\left(\left[\bar{\chi}_{\varphi}(\mathscr{T} \mathscr{F})\right]\right)=\mu \circ \psi_{\bowtie}([\mathrm{TF}])=\left[1 \otimes X_{1} \wedge X_{2} \wedge Y_{1}^{1} \wedge \cdots \wedge Y_{2}^{2}\right]
$$

thanks to the large kernel of (4.14). Hence the result follows since $\mu \circ \psi_{\triangleright}$ is an isomorphism on the level of cohomologies.

In the following we present the images of the cyclic cocycles $\mathscr{G} \mathscr{V}, \mathscr{R}_{1}, \mathscr{R}_{2}$, $\mathscr{R}_{3}$ and $\mathscr{R}_{4}$ under the characteristic map $\chi_{\varphi}: C^{\bullet}(\mathcal{K}, V) \rightarrow C^{\bullet+2}(\mathcal{A})$. We do not display the detailed account of the computation as it is lengthy and straightforward.

$$
\begin{aligned}
& \chi_{\varphi}(\mathscr{G} \mathscr{V})\left(a_{0} \otimes \cdots \otimes a_{5}\right)=\sum_{k=1}^{3} \sum_{\substack{1 \leq i, j \leq 2}} \sum_{\substack{\sigma, \gamma_{,} \\
\eta \in S_{2}}} 2 \cdot(-1)^{\sigma}(-1)^{\gamma}(-1)^{k-1} \\
& \left\{-\tau\left(a_{0} \delta_{i \eta(1)}^{i}\left(a_{1}\right) \delta_{j \eta(2)}^{j}\left(a_{2}\right) Y_{\mu^{k}(\sigma(1))}^{\mu^{k}(\sigma(2))}\left(a_{3}\right) Y_{\mu^{k}(\gamma(1))}^{\mu^{k}(\sigma(1))}\left(a_{4}\right) Y_{\mu^{k}(\gamma(2))}^{\mu^{k}(\sigma(1))}\left(a_{5}\right)\right)\right. \\
& +\tau\left(a_{0} \delta_{i \eta(1)}^{i}\left(a_{1}\right) Y_{\mu^{k}(\sigma(1))}^{\mu^{k}(\sigma(2))}\left(a_{2}\right) \delta_{j \eta(2)}^{j}\left(a_{3}\right) Y_{\mu^{k}(\gamma(1))}^{\mu^{k}(\sigma(1))}\left(a_{4}\right) Y_{\mu^{k}(\gamma(2))}^{\mu^{k}(\sigma(1))}\left(a_{5}\right)\right) \\
& -\tau\left(a_{0} \delta_{i \eta(1)}^{i}\left(a_{1}\right) Y_{\mu^{k}(\sigma(1))}^{\mu^{k}(\sigma(2))}\left(a_{2}\right) Y_{\mu^{k}(\gamma(1))}^{\mu^{k}(\sigma(1))}\left(a_{3}\right) \delta_{j \eta(2)}^{j}\left(a_{4}\right) Y_{\mu^{k}(\gamma(2))}^{\mu^{k}(\sigma(1))}\left(a_{5}\right)\right) \\
& +\tau\left(a_{0} \delta_{i \eta(1)}^{i}\left(a_{1}\right) Y_{\mu^{k}(\sigma(1))}^{\mu^{k}(\sigma(2))}\left(a_{2}\right) Y_{\mu^{k}(\gamma(1))}^{\mu^{k}(\sigma(1))}\left(a_{3}\right) Y_{\mu^{k}(\gamma(2))}^{\mu^{k}(\sigma(1))}\left(a_{4}\right) \delta_{j \eta(2)}^{j}\left(a_{5}\right)\right) \\
& -\tau\left(a_{0} Y_{\mu^{k}(\sigma(1))}^{\mu^{k}(\sigma(2))}\left(a_{1}\right) \delta_{i \eta(1)}^{i}\left(a_{2}\right) \delta_{j \eta(2)}^{j}\left(a_{3}\right) Y_{\mu^{k}(\gamma(1))}^{\mu^{k}(\sigma(1))}\left(a_{4}\right) Y_{\mu^{k}(\gamma(2))}^{\mu^{k}(\sigma(1))}\left(a_{5}\right)\right) \\
& +\tau\left(a_{0} Y_{\mu^{k}(\sigma(1))}^{\mu^{k}(\sigma(2))}\left(a_{1}\right) \delta_{i \eta(1)}^{i}\left(a_{2}\right) Y_{\mu^{k}(\gamma(1))}^{\mu^{k}(\sigma(1))}\left(a_{3}\right) \delta_{j \eta(2)}^{j}\left(a_{4}\right) Y_{\mu^{k}(\gamma(2))}^{\mu^{k}(\sigma(1))}\left(a_{5}\right)\right) \\
& -\tau\left(a_{0} Y_{\mu^{k}(\sigma(1))}^{\mu^{k}(\sigma(2))}\left(a_{1}\right) \delta_{i \eta(1)}^{i}\left(a_{2}\right) Y_{\mu^{k}(\gamma(1))}^{\mu^{k}(\sigma(1))}\left(a_{3}\right) Y_{\mu^{k}(\gamma(2))}^{\mu^{k}(\sigma(1))}\left(a_{4}\right) \delta_{j \eta(2)}^{j}\left(a_{5}\right)\right) \\
& -\tau\left(a_{0} Y_{\mu^{k}(\sigma(1))}^{\mu^{k}(\sigma(2))}\left(a_{1}\right) Y_{\mu^{k}(\gamma(1))}^{\mu^{k}(\sigma(1))}\left(a_{2}\right) \delta_{i \eta(1)}^{i}\left(a_{3}\right) \delta_{j \eta(2)}^{j}\left(a_{4}\right) Y_{\mu^{k}(\gamma(2))}^{\mu^{k}(\sigma(1))}\left(a_{5}\right)\right) \\
& +\tau\left(a_{0} Y_{\mu^{k}(\sigma(1))}^{\mu^{k}(\sigma(2))}\left(a_{1}\right) Y_{\mu^{k}(\gamma(1))}^{\mu^{k}(\sigma(1))}\left(a_{2}\right) \delta_{i \eta(1)}^{i}\left(a_{3}\right) Y_{\mu^{k}(\gamma(2))}^{\mu^{k}(\sigma(1))}\left(a_{4}\right) \delta_{j \eta(2)}^{j}\left(a_{5}\right)\right) \\
& \left.-\tau\left(a_{0} Y_{\mu^{k}(\sigma(1))}^{\mu^{k}(\sigma(2))}\left(a_{1}\right) Y_{\mu^{k}(\gamma(1))}^{\mu^{k}(\sigma(1))}\left(a_{2}\right) Y_{\mu^{k}(\gamma(2))}^{\mu^{k}(\sigma(1))}\left(a_{3}\right) \delta_{i \eta(1)}^{i}\left(a_{4}\right) \delta_{j \eta(2)}^{j}\left(a_{5}\right)\right)\right\} .
\end{aligned}
$$




$$
\begin{aligned}
\chi_{\varphi}\left(\mathscr{R}_{2}\right) & \left(a_{0} \otimes \cdots \otimes a_{3}\right)=\sum_{\sigma \in S_{2}}(-1)^{\sigma} \\
\{ & -\tau\left(a_{0} \delta_{2 \sigma(1)}^{1}\left(a_{1}\right) \delta_{1 \sigma(2)}^{2}\left(a_{2}\right) Y_{2}^{2}\left(a_{3}\right)\right) \\
& -\tau\left(a_{0} \delta_{1 \sigma(1)}^{2}\left(a_{1}\right) \delta_{2 \sigma(2)}^{1}\left(a_{2}\right) Y_{2}^{2}\left(a_{3}\right)\right)+\tau\left(a_{0} \delta_{2 \sigma(1)}^{1}\left(a_{1}\right) Y_{2}^{2}\left(a_{2}\right) \delta_{1 \sigma(2)}^{2}\left(a_{3}\right)\right) \\
& +\tau\left(a_{0} \delta_{1 \sigma(1)}^{2}\left(a_{1}\right) Y_{2}^{2}\left(a_{2}\right) \delta_{2 \sigma(2)}^{1}\left(a_{3}\right)\right)-\tau\left(a_{0} Y_{2}^{2}\left(a_{1}\right) \delta_{2 \sigma(1)}^{1}\left(a_{2}\right) \delta_{1 \sigma(2)}^{2}\left(a_{3}\right)\right) \\
& \left.-\tau\left(a_{0} Y_{2}^{2}\left(a_{1}\right) \delta_{1 \sigma(1)}^{2}\left(a_{2}\right) \delta_{2 \sigma(2)}^{1}\left(a_{3}\right)\right)\right\} .
\end{aligned}
$$

$\chi_{\varphi}\left(\mathscr{R}_{3}\right)\left(a_{0} \otimes a_{1} \otimes a_{2}\right)=\sum_{\substack{1 \leq i, j \\ j \leq 2}} \sum_{\sigma \in S_{2}} 2 \cdot(-1)^{\sigma} \tau\left(a_{0} \delta_{i \sigma(1)}^{i}\left(a_{1}\right) \delta_{j \sigma(2)}^{j}\left(a_{2}\right)\right)$.

$$
\begin{aligned}
& \chi_{\varphi}\left(\mathscr{R}_{1}\right)\left(a_{0} \otimes \cdots \otimes a_{5}\right)=\sum_{k=1}^{3} \sum_{\substack{\sigma, \gamma, \eta \in S_{2}}}(-1)^{\sigma}(-1)^{\gamma}(-1)^{k-1} \\
& \left\{-\tau\left(a_{0} \delta_{2 \eta(1)}^{1}\left(a_{1}\right) \delta_{1 \eta(2)}^{2}\left(a_{2}\right) Y_{\mu^{k}(\sigma(1))}^{\mu^{k}(\sigma(2))}\left(a_{3}\right) Y_{\mu^{k}(\gamma(1))}^{\mu^{k}(\sigma(1))}\left(a_{4}\right) Y_{\mu^{k}(\gamma(2))}^{\mu^{k}(\sigma(1))}\left(a_{5}\right)\right)\right. \\
& -\tau\left(a_{0} \delta_{1 \eta(1)}^{2}\left(a_{1}\right) \delta_{2 \eta(2)}^{1}\left(a_{2}\right) Y_{\mu^{k}(\sigma(1))}^{\mu^{k}(\sigma(2))}\left(a_{3}\right) Y_{\mu^{k}(\gamma(1))}^{\mu^{k}(\sigma(1))}\left(a_{4}\right) Y_{\mu^{k}(\gamma(2))}^{\mu^{k}(\sigma(1))}\left(a_{5}\right)\right) \\
& +\tau\left(a_{0} \delta_{2 \eta(1)}^{1}\left(a_{1}\right) Y_{\mu^{k}(\sigma(1))}^{\mu^{k}(\sigma(2))}\left(a_{2}\right) \delta_{1 \eta(2)}^{2}\left(a_{3}\right) Y_{\mu^{k}(\gamma(1))}^{\mu^{k}(\sigma(1))}\left(a_{4}\right) Y_{\mu^{k}(\gamma(2))}^{\mu^{k}(\sigma(1))}\left(a_{5}\right)\right) \\
& +\tau\left(a_{0} \delta_{1 \eta(1)}^{2}\left(a_{1}\right) Y_{\mu^{k}(\sigma(1))}^{\mu^{k}(\sigma(2))}\left(a_{2}\right) \delta_{2 \eta(2)}^{1}\left(a_{3}\right) Y_{\mu^{k}(\gamma(1))}^{\mu^{k}(\sigma(1))}\left(a_{4}\right) Y_{\mu^{k}(\gamma(2))}^{\mu^{k}(\sigma(1))}\left(a_{5}\right)\right) \\
& -\tau\left(a_{0} \delta_{2 \eta(1)}^{1}\left(a_{1}\right) Y_{\mu^{k}(\sigma(1))}^{\mu^{k}(\sigma(2))}\left(a_{2}\right) Y_{\mu^{k}(\gamma(1))}^{\mu^{k}(\sigma(1))}\left(a_{3}\right) \delta_{1 \eta(2)}^{2}\left(a_{4}\right) Y_{\mu^{k}(\gamma(2))}^{\mu^{k}(\sigma(1))}\left(a_{5}\right)\right) \\
& -\tau\left(a_{0} \delta_{1 \eta(1)}^{2}\left(a_{1}\right) Y_{\mu^{k}(\sigma(1))}^{\mu^{k}(\sigma(2))}\left(a_{2}\right) Y_{\mu^{k}(\gamma(1))}^{\mu^{k}(\sigma(1))}\left(a_{3}\right) \delta_{2 \eta(2)}^{1}\left(a_{4}\right) Y_{\mu^{k}(\gamma(2))}^{\mu^{k}(\sigma(1))}\left(a_{5}\right)\right) \\
& +\tau\left(a_{0} \delta_{2 \eta(1)}^{1}\left(a_{1}\right) Y_{\mu^{k}(\sigma(1))}^{\mu^{k}(\sigma(2))}\left(a_{2}\right) Y_{\mu^{k}(\gamma(1))}^{\mu^{k}(\sigma(1))}\left(a_{3}\right) Y_{\mu^{k}(\gamma(2))}^{\mu^{k}(\sigma(1))}\left(a_{4}\right) \delta_{1 \eta(2)}^{2}\left(a_{5}\right)\right) \\
& +\tau\left(a_{0} \delta_{1 \eta(1)}^{2}\left(a_{1}\right) Y_{\mu^{k}(\sigma(1))}^{\mu^{k}(\sigma(2))}\left(a_{2}\right) Y_{\mu^{k}(\gamma(1))}^{\mu^{k}(\sigma(1))}\left(a_{3}\right) Y_{\mu^{k}(\gamma(2))}^{\mu^{k}(\sigma(1))}\left(a_{4}\right) \delta_{2 \eta(2)}^{1}\left(a_{5}\right)\right) \\
& -\tau\left(a_{0} Y_{\mu^{k}(\sigma(1))}^{\mu^{k}(\sigma(2))}\left(a_{1}\right) \delta_{2 \eta(1)}^{1}\left(a_{2}\right) \delta_{1 \eta(2)}^{2}\left(a_{3}\right) Y_{\mu^{k}(\gamma(1))}^{\mu^{k}(\sigma(1))}\left(a_{4}\right) Y_{\mu^{k}(\gamma(2))}^{\mu^{k}(\sigma(1))}\left(a_{5}\right)\right) \\
& -\tau\left(a_{0} Y_{\mu^{k}(\sigma(1))}^{\mu^{k}(\sigma(2))}\left(a_{1}\right) \delta_{1 \eta(1)}^{2}\left(a_{2}\right) \delta_{2 \eta(2)}^{1}\left(a_{3}\right) Y_{\mu^{k}(\gamma(1))}^{\mu^{k}(\sigma(1))}\left(a_{4}\right) Y_{\mu^{k}(\gamma(2))}^{\mu^{k}(\sigma(1))}\left(a_{5}\right)\right) \\
& +\tau\left(a_{0} Y_{\mu^{k}(\sigma(1))}^{\mu^{k}(\sigma(2))}\left(a_{1}\right) \delta_{2 \eta(1)}^{1}\left(a_{2}\right) Y_{\mu^{k}(\gamma(1))}^{\mu^{k}(\sigma(1))}\left(a_{3}\right) \delta_{1 \eta(2)}^{2}\left(a_{4}\right) Y_{\mu^{k}(\gamma(2))}^{\mu^{k}(\sigma(1))}\left(a_{5}\right)\right) \\
& +\tau\left(a_{0} Y_{\mu^{k}(\sigma(1))}^{\mu^{k}(\sigma(2))}\left(a_{1}\right) \delta_{1 \eta(1)}^{2}\left(a_{2}\right) Y_{\mu^{k}(\gamma(1))}^{\mu^{k}(\sigma(1))}\left(a_{3}\right) \delta_{2 \eta(2)}^{1}\left(a_{4}\right) Y_{\mu^{k}(\gamma(2))}^{\mu^{k}(\sigma(1))}\left(a_{5}\right)\right)
\end{aligned}
$$




$$
\begin{aligned}
& -\tau\left(a_{0} Y_{\mu^{k}(\sigma(1))}^{\mu^{k}(\sigma(2))}\left(a_{1}\right) \delta_{2 \eta(1)}^{1}\left(a_{2}\right) Y_{\mu^{k}(\gamma(1))}^{\mu^{k}(\sigma(1))}\left(a_{3}\right) Y_{\mu^{k}(\gamma(2))}^{\mu^{k}(\sigma(1))}\left(a_{4}\right) \delta_{1 \eta(2)}^{2}\left(a_{5}\right)\right) \\
& -\tau\left(a_{0} Y_{\mu^{k}(\sigma(1))}^{\mu^{k}(\sigma(2))}\left(a_{1}\right) \delta_{1 \eta(1)}^{2}\left(a_{2}\right) Y_{\mu^{k}(\gamma(1))}^{\mu^{k}(\sigma(1))}\left(a_{3}\right) Y_{\mu^{k}(\gamma(2))}^{\mu^{k}(\sigma(1))}\left(a_{4}\right) \delta_{2 \eta(2)}^{1}\left(a_{5}\right)\right) \\
& -\tau\left(a_{0} Y_{\mu^{k}(\sigma(1))}^{\mu^{k}(\sigma(2))}\left(a_{1}\right) Y_{\mu^{k}(\gamma(1))}^{\mu^{k}(\sigma(1))}\left(a_{2}\right) \delta_{2 \eta(1)}^{1}\left(a_{3}\right) \delta_{1 \eta(2)}^{2}\left(a_{4}\right) Y_{\mu^{k}(\gamma(2))}^{\mu^{k}(\sigma(1))}\left(a_{5}\right)\right) \\
& -\tau\left(a_{0} Y_{\mu^{k}(\sigma(1))}^{\mu^{k}(\sigma(2))}\left(a_{1}\right) Y_{\mu^{k}(\gamma(1))}^{\mu^{k}(\sigma(1))}\left(a_{2}\right) \delta_{1 \eta(1)}^{2}\left(a_{3}\right) \delta_{2 \eta(2)}^{1}\left(a_{4}\right) Y_{\mu^{k}(\gamma(2))}^{\mu^{k}(\sigma(1))}\left(a_{5}\right)\right)
\end{aligned}
$$

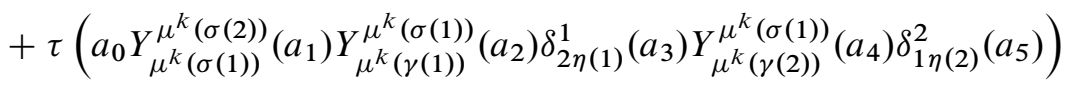

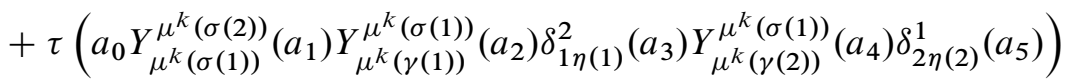

$$
\begin{aligned}
& -\tau\left(a_{0} Y_{\mu^{k}(\sigma(1))}^{\mu^{k}(\sigma(2))}\left(a_{1}\right) Y_{\mu^{k}(\gamma(1))}^{\mu^{k}(\sigma(1))}\left(a_{2}\right) Y_{\mu^{k}(\gamma(2))}^{\mu^{k}(\sigma(1))}\left(a_{3}\right) \delta_{2 \eta(1)}^{1}\left(a_{4}\right) \delta_{1 \eta(2)}^{2}\left(a_{5}\right)\right) \\
& \left.-\tau\left(a_{0} Y_{\mu^{k}(\sigma(1))}^{\mu^{k}(\sigma(2))}\left(a_{1}\right) Y_{\mu^{k}(\gamma(1))}^{\mu^{k}(\sigma(1))}\left(a_{2}\right) Y_{\mu^{k}(\gamma(2))}^{\mu^{k}(\sigma(1))}\left(a_{3}\right) \delta_{1 \eta(1)}^{2}\left(a_{4}\right) \delta_{2 \eta(2)}^{1}\left(a_{5}\right)\right)\right\} .
\end{aligned}
$$

Finally,

$$
\begin{aligned}
\chi_{\varphi}\left(\mathscr{R}_{4}\right) & \left(a_{0} \otimes a_{1} \otimes a_{2}\right) \\
& =\varphi\left(c_{2} \otimes a_{0} \otimes a_{1} \otimes a_{2}\right) \\
& =\sum_{\sigma \in S_{2}}(-1)^{\sigma}\left\{\tau\left(a_{0} \delta_{2 \sigma(1)}^{1}\left(a_{1}\right) \delta_{1 \sigma(2)}^{2}\left(a_{2}\right)\right)+\tau\left(a_{0} \delta_{1 \sigma(1)}^{2}\left(a_{1}\right) \delta_{2 \sigma(2)}^{1}\left(a_{2}\right)\right)\right\} .
\end{aligned}
$$

Remark 4.6. One knows that the characteristic map $\chi_{\tau}: C^{\bullet}\left(\mathcal{H}, \mathbb{C}_{\delta}\right) \rightarrow C^{\bullet}(\mathcal{A})$ is injective [2]. Since $\chi_{\varphi}(\mathscr{T} \mathscr{F}), \chi_{\varphi}(\mathscr{G} \mathscr{V}), \chi_{\varphi}\left(\mathscr{R}_{1}\right), \chi_{\varphi}\left(\mathscr{R}_{2}\right), \chi_{\varphi}\left(\mathscr{R}_{3}\right)$, and $\chi_{\varphi}\left(\mathscr{R}_{4}\right)$, are all in the range of $\chi_{\tau}$, as a byproduct of our study in this paper, one calculates cyclic cocycles representing a basis for $H P\left(\mathcal{H}, \mathbb{C}_{\delta}\right)$.

\section{References}

[1] A. Connes, Noncommutative geometry, Academic Press Inc., San Diego, CA, 1994. Zbl 0818.46076 MR 1303779

[2] A. Connes and H. Moscovici, Hopf algebras, cyclic cohomology and the transverse index theorem, Comm. Math. Phys. 198 (1998), no. 1, 199-246. Zbl 0940.58005 MR 1657389

[3] A. Connes and H. Moscovici, Background independent geometry and Hopf cyclic cohomology, 2005.

[4] C. Godbillon, Cohomologies d'algèbres de Lie de champs de vecteurs formels, Séminaire Bourbaki, 25ème année (1972/1973), Exp. No. 421. Lecture Notes in Math., 383, 69-87. Springer, Berlin, 1974. Zbl 0296.17010 MR 418112 
[5] P. M. Hajac, M. Khalkhali, B. Rangipour and Y. Sommerhäuser, Hopf-cyclic homology and cohomology with coefficients, C. R. Math. Acad. Sci. Paris 338 (2004), no. 9, 667-672. Zbl 1064.16006 MR 2065371

[6] M. Khalkhali and B. Rangipour, Invariant cyclic homology, K-Theory 28 (2003), no. 2, 183-205. Zbl 1028.58006 MR 1995876

[7] M. Khalkhali and B. Rangipour, On the generalized cyclic Eilenberg-Zilber theorem, Canad. Math. Bull. 47 (2004), no. 1, 38-48. Zbl 1048.19004 MR 2032267

[8] M. Khalkhali and B. Rangipour, Cup products in Hopf-cyclic cohomology, C. R. Math. Acad. Sci. Paris 340 (2005), no. 1, 9-14. Zbl 1063.16011 MR 2112033

[9] H. Moscovici and B. Rangipour, Cyclic cohomology of Hopf algebras of transverse symmetries in codimension 1, Adv. Math. 210 (2007), no. 1, 323374. Zbl 1124.16008 MR 2298827

[10] H. Moscovici and B. Rangipour, Hopf algebras of primitive Lie pseudogroups and Hopf cyclic cohomology, Adv. Math. 220 (2009), no. 3, 706-790. Zbl 1189.58008 MR 2483227

[11] H. Moscovici and B. Rangipour, Hopf cyclic cohomology and transverse characteristic classes, Adv. Math. 227 (2011), no. 1, 654-729. Zbl 1268.16010 MR 2782206

[12] B. Rangipour, Cup products in Hopf cyclic cohomology via cyclic modules, Homology, Homotopy Appl. 10 (2008), no. 2, 273-286. Zbl 1179.16003 MR 2475613

[13] B. Rangipour and S. Sütlü, Cyclic cohomology of Lie algebras, Documenta Math. 17 (2012), 483-515. Zbl 1307.17024 MR 2992958

Received 19 February, 2014

B. Rangipour, Department of Mathematics and Statistics, University of New Brunswick, P.O. Box 4400, Fredericton, New Brunswick E3B 5A3, Canada

E-mail: bahram@unb.ca

S. Sütlü, Işık University, Department of Mathematics, 34980, Şile - İstanbul, Turkey

E-mail: serkan.sutlu@isikun.edu.tr 